\title{
Well-posedness, stability and invariance results for a class of multivalued Lur'e dynamical systems
}

\author{
Bernard Brogliato ${ }^{\mathrm{a}, *}$, Daniel Goeleven ${ }^{\mathrm{b}}$ \\ a INRIA, BIPOP project-team, ZIRST Montbonnot, 655 avenue de l'Europe, 38334 Saint Ismier, France \\ ${ }^{\mathrm{b}}$ PIMENT, Université de La Réunion, Saint-Denis, 97400, France
}

\begin{abstract}
This paper analyzes the existence and uniqueness issues in a class of multivalued Lur'e systems, where the multivalued part is represented as the subdifferential of some convex, proper, lower semicontinuous function. Through suitable transformations the system is recast into the framework of dynamic variational inequalities and the well-posedness (existence and uniqueness of solutions) is proved. Stability and invariance results are also studied, together with the property of continuous dependence on the initial conditions. The problem is motivated by practical applications in electrical circuits containing electronic devices with nonsmooth multivalued voltage/current characteristics, and by state observer design for multivalued systems.
\end{abstract}

\section{Introduction}

Lur'e systems, which consist of a linear time-invariant system in negative feedback with a static nonlinearity satisfying a sector condition, have received a considerable interest in the applied mathematics and control literature, due to their broad interest (see [1] for a survey). More recently the case where the nonlinearity is a maximal monotone map has been studied [2]. The maximal monotonicity allows one to consider unbounded sectors $[0,+\infty]$ and nonsmooth set-valued nonlinearities. So-called linear complementarity systems can be recast into Lur'e systems, where the feedback nonlinearity takes the form of a set of complementarity conditions between two slack variables [3-5]. One of these slack variables may be interpreted as a Lagrange multiplier $\lambda$, while the other one usually takes the form $y=C x+D \lambda$. More general piecewise linear nonlinearities have been considered in [6,7]. As pointed out in [2] there exists a close relationship between some complementarity systems and differential inclusions with maximal monotone right-hand sides, in particular inclusions into normal cones to convex sets (which are in turn equivalent to dynamical variational inequalities of the first kind). Particular cases have been investigated in [8-10]. All these works are however restricted to the case where $D=0$, except [7] where affine complementarity systems are considered. In this paper, we extend the works in $[8,9]$ to the case where $D \neq 0$, i.e. there exists a feedthrough matrix in the linear part of the system. Moreover the nonlinearities which we consider are much more general than complementarity conditions between $y$ and $\lambda$ (i.e. $y \geq 0, \lambda \geq 0, y^{T} \lambda=0$ ) and the considered systems may be written equivalently as dynamical variational inequalities of the second kind. Such an extension may be important in practice (for instance electrical circuits with ideal diodes and transistors usually yield systems with a nonzero feedthrough matrix $D$, possibly positive semi-definite and non-symmetric). Observer synthesis for set-valued systems is also an important application $[11,12]$. This work may also be seen as the continuation of previous efforts to study the relationships

\footnotetext{
* Corresponding author. Tel.: +33 476615393; fax: +33 476615252.

E-mail addresses: Bernard.Brogliato@inrialpes.fr (B. Brogliato), Daniel.Goeleven@univ-reunion.fr (D. Goeleven).
} 
between various types of differential inclusions, complementarity systems, projected systems in finite dimensions [10,13-15].

The paper is organized as follows: In Section 2 the dynamical system is presented, and its well-posedness is studied in Section 3. In Section 4 the stability properties are studied, and an invariance result is presented in Section 5. Conclusions end the paper in Section 6.

Notations: Let $f: \mathbb{R}^{n} \rightarrow \mathbb{R} \cup\{+\infty\}$ be a proper convex and lower semicontinuous function, we denote by dom $(f):=\{x \in$ $\left.\mathbb{R}^{n}: f(x)<+\infty\right\}$ the domain of the function $f(\cdot)$. Recall that the Fenchel transform $f^{*}(\cdot)$ of $f(\cdot)$ is the proper, convex and lower semicontinuous function defined by

$$
\left(\forall z \in \mathbb{R}^{n}\right): f^{*}(z)=\sup _{x \in \operatorname{dom}(f)}\{\langle x, z\rangle-f(x)\} .
$$

The subdifferential $\partial f(x)$ of $f(\cdot)$ at $x \in \mathbb{R}^{n}$ is defined by

$$
\partial f(x)=\left\{\omega \in \mathbb{R}^{n}: f(v)-f(x) \geq\langle\omega, v-x\rangle, \forall v \in \mathbb{R}^{n}\right\},
$$

where $\langle\cdot, \cdot\rangle$ denotes the usual scalar product in $\mathbb{R}^{n}$, i.e. $\langle y, z\rangle=y^{T} z$ for any vectors $y$ and $z$ of $\mathbb{R}^{n}$. We denote by Dom $(\partial f):=$ $\left\{x \in \mathbb{R}^{n}: \partial f(x) \neq \emptyset\right\}$ the domain of the subdifferential operator $\partial f: \mathbb{R}^{n} \rightarrow \mathbb{R}^{n}$. Let $x_{0}$ be any element in the domain $\operatorname{dom}(f)$ of $f(\cdot)$, the recession function $f_{\infty}(\cdot)$ of $f(\cdot)$ is defined by

$$
\left(\forall x \in \mathbb{R}^{n}\right): f_{\infty}(x)=\lim _{\lambda \rightarrow+\infty} \frac{1}{\lambda} f\left(x_{0}+\lambda x\right) .
$$

The function $f_{\infty}: \mathbb{R}^{n} \rightarrow \mathbb{R} \cup\{+\infty\}$ is a proper convex and lower semicontinuous function which describes the asymptotic behavior of $f(\cdot)$. For a nonempty closed and convex set $K \subset \mathbb{R}^{n}$, the dual cone of $K$ is the nonempty closed convex cone $K^{\star}$ defined by

$$
K^{\star}:=\left\{w \in \mathbb{R}^{n}:\langle w, v\rangle \geq 0, \forall v \in K\right\},
$$

while the polar cone $K^{0}=-K^{\star}$. Let $x_{0}$ be any element in $K$, the recession cone of $K$ is defined by

$$
K_{\infty}=\bigcap_{\lambda>0} \frac{1}{\lambda}\left(K-x_{0}\right) .
$$

The set $K_{\infty}$ is a nonempty closed convex cone that is described in terms of the directions which recede from $K$. When $K$ is a cone then $K_{\infty}=K$. The relative interior of a set $K$ is denoted as rint $(K)$, and its closure as $\bar{K}$. Let $M \in \mathbb{R}^{m \times n}$ be a given matrix, we denote by $\operatorname{ker}(M)$ the kernel of $M$ and by $\mathcal{R}(M)$ the range of $M . M \geq 0$ means that $M$ is positive semidefinite, $M>0$ means that it is positive definite.

\section{The multivalued Lur'e system}

Let $A \in \mathbb{R}^{n \times n}, B \in \mathbb{R}^{n \times p}, C \in \mathbb{R}^{p \times n}, D \in \mathbb{R}^{p \times p}$ be given matrices, $f \in C^{0}\left(\mathbb{R}_{+} ; \mathbb{R}\right)$ such that $f^{\prime} \in L_{\text {loc }}^{1}\left(\mathbb{R}_{+} ; \mathbb{R}^{n}\right)$ and $\varphi_{i}: \mathbb{R} \rightarrow \mathbb{R} \cup\{+\infty\}(1 \leq i \leq p)$ given proper convex and lower semicontinuous functions. Let $x_{0} \in \mathbb{R}^{n}$ be some initial condition, we consider the problem: Find $x \in C^{0}\left(\mathbb{R}_{+} ; \mathbb{R}^{n}\right)$ such that $x^{\prime} \in L_{\text {loc }}^{\infty}\left(\mathbb{R}_{+} ; \mathbb{R}^{n}\right)$ and $x$ right-differentiable on $\mathbb{R}_{+}$, $\lambda \in C^{0}\left(\mathbb{R}_{+} ; \mathbb{R}^{p}\right)$ and $y \in C^{0}\left(\mathbb{R}_{+} ; \mathbb{R}^{p}\right)$ satisfying the nonsmooth dynamical system $\operatorname{NSDS}\left(A, B, C, D, f, \varphi_{1}, \ldots, \varphi_{p}, x_{0}\right)$ :

$$
\left\{\begin{array}{l}
x(0)=x_{0} \text { a.e. } t \geq 0 \\
x^{\prime}(t)=A x(t)+B \lambda(t)+f(t) \quad \text { for all } t \geq 0 \\
y(t)=C x(t)+D \lambda(t) \\
\lambda_{1}(t) \in-\partial \varphi_{1}\left(y_{1}(t)\right) \\
\lambda_{2}(t) \in-\partial \varphi_{2}\left(y_{2}(t)\right) \\
\vdots \\
\lambda_{p}(t) \in-\partial \varphi_{p}\left(y_{p}(t)\right) .
\end{array}\right.
$$

The system is therefore in the canonical absolute stability form since it is the negative feedback interconnection of a linear invariant system $(A, B, C, D)$ (with "input" $\lambda$, "output" $y$ and external excitation $f(\cdot)$ ) with a static multivalued nonlinearity (with "input" $y$ and "output" $-\lambda$ ). In $[8,9]$ it was considered that $D=0$. As we shall see next the case $D \neq 0$ complicates the analysis. It is noteworthy that one may have $p>n$, which is crucial because $\lambda$ is not a control input and $p$ may in applications be very large. Physical examples are given later in the paper. It is assumed in this paper that the "output" $y$ does not depend explicitly on time. If this is the case the results of this paper do not apply because one has to resort to the perturbed Moreau's sweeping process to derive well-posedness results, see [16].

Let us set $\lambda=\left(\lambda_{1} \cdots \lambda_{p}\right)^{T}, \Phi(\cdot)=\varphi_{1}(\cdot)+\cdots+\varphi_{p}(\cdot)$, and $M \in \mathbb{R}^{p \times p}$ is an invertible matrix. One may consider a slightly more general version of the Lur'e system (2) as:

$$
\left\{\begin{array}{l}
x(0)=x_{0} \\
x^{\prime}(t)=A x(t)+B \lambda(t)+f(t) \\
\lambda(t) \in-M \partial \Phi(y(t)) .
\end{array}\right.
$$




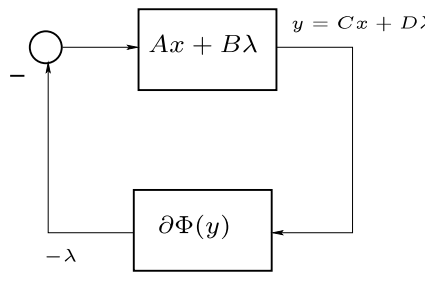

a

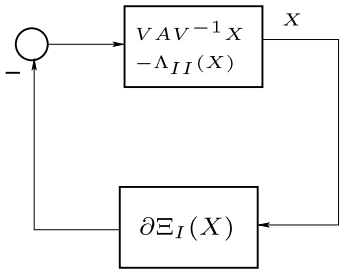

$\mathrm{b}$

Fig. 1. Lur'e system transformation.

Denoting $\bar{\lambda}=M^{-1} \lambda, \bar{B}=B M, \bar{D}=D M$, (3) is equivalently rewritten as:

$$
\left\{\begin{array}{l}
x(0)=x_{0} \\
x^{\prime}(t)=A x(t)+\bar{B} \bar{\lambda}(t)+f(t) \\
\bar{\lambda}(t) \in-\partial \Phi(C x(t)+\bar{D} \bar{\lambda}) .
\end{array}\right.
$$

Therefore the transformed system (4) possesses the same structure as the system in (2). The Lur'e system (2) can be represented as in Fig. 1(a).

Finally as will appear clearly later, all the existence and uniqueness of solutions results which are derived in this paper (Section 3) also hold when the linear term $A x$ is replaced by a Lipschitz continuous mapping $A(x)$. For the sake of clarity of the presentation the linear case $A x$ is kept all through the paper, for the well-posedness and the stability analysis.

\section{Well-posedness analysis}

In this section the existence and uniqueness of solutions will be shown first by using a version of Kato's theorem, second via maximal monotone operators. Examples coming from electrical circuits and state observer design are provided to illustrate the theoretical developments.

\subsection{Well-posedness by Kato's theorem}

In the remainder of this section we shall apply some transformations to the Lur'e system so that its well-posedness can be analyzed.

\subsubsection{System's transformations}

Let us set

$$
(\forall z \in \mathbb{R}): \varphi_{i}^{*,-}(z):=\varphi_{i}^{*}(-z) .
$$

Assumption 1. We assume the existence of $z_{0, i} \in \mathbb{R}$ at which $\varphi_{i}^{*,-}(\cdot)$ is continuous.

Assumption 1 is a simple qualitative condition that is required to ensure that (see [17]):

$$
(\forall z \in \mathbb{R}): \partial \varphi_{i}^{*,-}(z)=-\partial \varphi_{i}^{*}(-z) .
$$

Then

$$
\lambda_{i} \in-\partial \varphi_{i}\left(y_{i}\right) \Leftrightarrow y_{i} \in \partial \varphi_{i}^{*}\left(-\lambda_{i}\right)=-\left[-\partial \varphi_{i}^{*}\left(-\lambda_{i}\right)\right]=-\partial \varphi_{i}^{*,-}\left(\lambda_{i}\right) .
$$

Let us now denote by $p_{I}$ (and set $p_{I I}=p-p_{I}$ ) the largest integer such that the matrix $D$ can be written as follows:

$$
D=\left(\begin{array}{cc}
0_{p_{I} \times p_{I}} & 0_{p_{I I} \times p_{I}} \\
0_{p_{I} \times p_{I I}} & D_{I I}
\end{array}\right)
$$

with $D_{I I} \neq 0_{p_{I I} \times p_{I I}}$. In using this notation, we suppose by convention that $p_{I}=0$ (resp. $\left.p_{I I}=0\right)$ means that the terms indexed by $I$ (resp. II) are useless and not considered. So, if $p_{I}=0$ (resp. $p_{I I}=0$ ) then $D \equiv D_{I I}$ (resp. $D \equiv 0_{p \times p}$ ). For $z \in \mathbb{R}^{p}$, we set

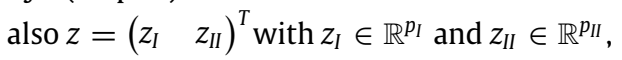

$$
B=\left(\begin{array}{ll}
B^{I} & B^{I I}
\end{array}\right), \quad C=\left(\begin{array}{c}
C_{I} \\
C_{I I}
\end{array}\right)
$$

with $B^{I} \in \mathbb{R}^{n \times p_{I}}, B^{I I} \in \mathbb{R}^{n \times p_{I I}}, C_{I} \in \mathbb{R}^{p_{I} \times n}$ and $C_{I I} \in \mathbb{R}^{p_{I I} \times n}$. Finally, we set

$$
\left(\forall y \in \mathbb{R}^{p_{I}}\right): \Phi_{I}(y):=\varphi_{1}\left(y_{1}\right)+\varphi_{2}\left(y_{2}\right)+\cdots+\varphi_{p_{I}}\left(y_{p_{I}}\right)
$$


and

$$
\left(\forall y \in \mathbb{R}^{p_{I I}}\right): \Phi_{I I}(y):=\varphi_{p_{I}+1}\left(y_{1}\right)+\varphi_{p_{I}+2}\left(y_{2}\right)+\cdots+\varphi_{p_{I I}}\left(y_{p_{I I}}\right) .
$$

We have:

$$
\left(\forall z \in \mathbb{R}^{p_{I I}}\right): \Phi_{I I}^{*}(z)=\varphi_{p_{I}+1}^{*}\left(z_{1}\right)+\varphi_{p_{I}+2}^{*}\left(z_{2}\right)+\cdots+\varphi_{p_{I I}}^{*}\left(z_{p_{I I}}\right) .
$$

We set

$$
\left(\forall z \in \mathbb{R}^{p_{I I}}\right): \Phi_{I I}^{*,-}(z):=\Phi_{I I}^{*}(-z) .
$$

We note also that Assumption 1 ensures that:

$$
\left(\forall z \in \mathbb{R}^{p_{I I}}\right): \partial \Phi_{I I}^{*,-}(z)=-\partial \Phi_{I I}^{*}(-z) .
$$

We also set:

$$
\left(\forall x \in \mathbb{R}^{p}\right): \Phi(x)=\Phi_{I}(x)+\Phi_{I I}(x)
$$

and

$$
\left(\forall x \in \mathbb{R}^{p}\right): \Phi^{*,-}(x)=\Phi^{*}(-x) .
$$

Assumption 1 guarantees that

$\left(\forall x \in \mathbb{R}^{p}\right): \partial \Phi^{*,-}(x)=-\partial \Phi^{*}(-x)$.

It follows that the system

$$
\left\{\begin{array}{l}
\lambda_{1}(t) \in-\partial \varphi_{1}\left(y_{1}(t)\right) \\
\lambda_{2}(t) \in-\partial \varphi_{2}\left(y_{2}(t)\right) \\
\vdots \\
\lambda_{p}(t) \in-\partial \varphi_{p}\left(y_{p}(t)\right)
\end{array}\right.
$$

can be written equivalently as:

$$
\left\{\begin{array}{l}
\lambda_{I}(t) \in-\partial \Phi_{I}\left(y_{I}(t)\right) \\
\lambda_{I I}(t) \in-\partial \Phi_{I I}\left(y_{I I}(t)\right)
\end{array}\right.
$$

or as:

$$
\left\{\begin{array}{l}
\lambda_{I}(t) \in-\partial \Phi_{I}\left(y_{I}(t)\right) \\
y_{I I}(t) \in-\partial \Phi_{I I}^{*-}\left(\lambda_{I I}(t)\right) .
\end{array}\right.
$$

Using these notations, we see that the system $\operatorname{NSDS}\left(A, B, C, D, f, \varphi_{1}, \ldots, \varphi_{p}, x_{0}\right)$ reduces to the system:

$$
\left\{\begin{array}{l}
x(0)=x_{0} \text { a.e. } t \geq 0 \\
x^{\prime}(t)=A x(t)+B^{I} \lambda_{I}(t)+B^{I I} \lambda_{I I}(t)+f(t) \quad \text { for all } t \geq 0 \\
y_{I}(t)=C_{I} x(t) \\
\lambda_{I}(t) \in-\partial \Phi_{I}\left(y_{I}(t)\right) \\
y_{I I}(t)=C_{I I} x(t)+D_{I I} \lambda_{I I}(t) \\
y_{I I}(t) \in-\partial \Phi_{I I}^{*,-}\left(\lambda_{I I}(t)\right) .
\end{array}\right.
$$

The feedback nonlinearity is therefore split into two main parts: one part indexed by $I$ is multivalued, the other part indexed by II will be shown under certain conditions to be single-valued.

Remark 1. The case $p_{I}=n$ (i.e. $D=0_{n \times n}$ ) has been the object of specific papers, see $[8,9,18]$. The complementarity problem (i.e. $\left.\varphi_{i}(\cdot)=\Psi_{\mathbb{R}_{+}}(\cdot) \forall i \in\{1, \ldots, n\}\right)$, has also been the object of various papers $[3,4,19,20]$.

Assumption 2 (If $p_{I} \geq 1$ ). There exists a symmetric and invertible matrix $W \in \mathbb{R}^{n \times n}$ such that:

$$
W^{2} B^{I}=C_{I}^{T} .
$$

We set:

$$
V= \begin{cases}W & \text { if } p_{I} \geq 1 \\ I & \text { if } p_{I}=0\end{cases}
$$

and

$$
\left(\forall w \in \mathbb{R}^{p_{I}}\right): \Xi_{I}(w)= \begin{cases}\Phi_{I}\left(C_{I} V^{-1} w\right) & \text { if } p_{I} \geq 1 \\ 0 & \text { if } p_{I}=0 .\end{cases}
$$

Notice that by [21, Exercise 1.40, Proposition 1.39] the function $\Xi_{I}(\cdot)=\Phi \circ C_{I} V^{-1}(\cdot)$ is lower semicontinuous, proper, convex. 
Assumption 3 (If $p_{I} \geq 1$ ). There exists a point $w_{0}$ in $\mathbb{R}^{p_{I}}$ at which $\Xi_{I}(\cdot)$ is continuous.

Assumptions 2 and 3 ensure in case $p_{I} \geq 1$ that

$\left(\forall w \in \mathbb{R}^{p_{I}}\right): \partial \Xi_{I}(w)=V^{-T} C_{I}^{T} \partial \Phi_{I}\left(C_{I} V^{-1} w\right)=V^{-1} C_{I}^{T} \partial \Phi_{I}\left(C_{I} V^{-1} w\right)$.

The multivalued mapping $\Xi_{I}(\cdot)$ is maximal monotone, being the subdifferential of a convex, proper, lower semicontinuous function. Let us now set:

$$
\left(\forall x \in \mathbb{R}^{n}\right): \Lambda_{I I}(x):= \begin{cases}V B^{I I}\left(D_{I I}+\partial \Phi_{I I}^{*,-}\right)^{-1}\left(-C_{I I} V^{-1} x\right) & \text { if } p_{I I} \geq 1 \\ 0 & \text { if } p_{I I}=0\end{cases}
$$

We suppose also the following:

Assumption 4 (If $p_{I I} \geq 1$ ). The operator $\Lambda_{I I}: \mathbb{R}^{n} \rightarrow \mathbb{R}^{n}: x \mapsto \Lambda_{I I}(x)$ is well-defined, single-valued and Lipschitz continuous.

Recalling that

$$
D_{I I} z-q \in-\partial \Phi_{I I}^{*,-}(z) \Leftrightarrow q \in\left(D_{I I}+\partial \Phi_{I I}^{*,-}\right)(z) \Leftrightarrow z \in\left(D_{I I}+\partial \Phi_{I I}^{*,-}\right)^{-1}(q),
$$

we note that Assumption 4 (in case $p_{I I} \geq 1$ ) requires that for all $q \in \mathbb{R}^{p_{I I}}$, there exists at least one $z(q) \in \mathbb{R}^{p_{I I}}$ such that

$$
\left\langle D_{I I} z-q, v-z\right\rangle+\Phi_{I I}^{*,-}(v)-\Phi_{I I}^{*,-}(z) \geq 0, \quad \forall v \in \mathbb{R}^{p_{I I}},
$$

and there exists a constant $K>0$ such that for all $x_{1}, x_{2} \in \mathbb{R}^{n}$ and $z_{1} \in\left(D_{I I}+\partial \Phi_{I I}^{*,-}\right)^{-1}\left(-C_{I I} V^{-1} x_{1}\right), z_{2} \in\left(D_{I I}+\right.$ $\left.\partial \Phi_{I I}^{*,-}\right)^{-1}\left(-C_{I I} V^{-1} x_{2}\right)$ :

$$
\left\|V B^{I I} z_{1}-V B^{I I} z_{2}\right\| \leq K\left\|x_{1}-x_{2}\right\| .
$$

The solvability of the variational inequality in (15) ensures that

$$
\left(\forall x \in \mathbb{R}^{n}\right): V B^{I I}\left(D_{I I}+\partial \Phi_{I I}^{*,-}\right)^{-1}\left(-C_{I I} V^{-1} x\right) \neq \emptyset
$$

while the condition in (16) guarantees that if $x \in \mathbb{R}^{n}$ and $z_{1}, z_{2} \in\left(D_{I I}+\partial \Phi_{I I}^{*,-}\right)^{-1}\left(-C_{I I} V^{-1} x\right)$ then $\left\|z_{1}-z_{2}\right\| \leq 0$ and thus $z_{1}=z_{2}$. It results that the operator $\Lambda_{I I}(\cdot)$ is single-valued. The Lipschitz continuity of $\Lambda_{I I}(\cdot)$ is then also a direct consequence of (16).

Conditions on the matrix $D_{I I}$ and on the function $\Phi_{I I}^{*,-}(\cdot)$ ensuring that Assumption 4 holds will be discussed in the following section.

The problem $\operatorname{NSDS}\left(A, B, C, D, f, \varphi_{1}, \ldots, \varphi_{p}, x_{0}\right)$ can be reduced, by setting $X(t)=V x(t)(\forall t \geq 0)$, to the following dynamical variational inequality problem: Find $X \in C^{0}\left(\mathbb{R}_{+} ; \mathbb{R}^{n}\right)$ such that $X^{\prime} \in L_{\text {loc }}^{\infty}\left(\mathbb{R}_{+} ; \mathbb{R}^{n}\right)$ and $X$ right-differentiable on $\mathbb{R}_{+}$such that $X(0)=V x_{0}$ and satisfying for a.e. $t \geq 0$ the variational inequality:

$$
\left\langle X^{\prime}(t)-V A V^{-1} X(t)-\Lambda_{I I}(X(t))-V f(t), v-X(t)\right\rangle+\Xi_{I}(v)-\Xi_{I}(X(t)) \geq 0, \quad \forall v \in \mathbb{R}^{n}
$$

which we may name a dynamical variational inequality of the second kind. Indeed, let us here write the details in case $p_{I} \geq 1$ and $p_{I I} \geq 1$. It is clear that

$$
x(0)=x_{0} \Leftrightarrow V x(0)=V x_{0} \Leftrightarrow X(0)=V x_{0}
$$

and

$$
\begin{aligned}
& \left\{\begin{array}{l}
x^{\prime}(t)=A x(t)+B^{I} \lambda_{I}(t)+B^{I I} \lambda_{I I}(t)+f(t) \\
y_{I}(t)=C_{I} x(t) \\
\lambda_{I}(t) \in-\partial \Phi_{I}\left(y_{I}(t)\right) \\
y_{I I}(t)=C_{I I} x(t)+D_{I I} \lambda_{I I}(t) \\
y_{I I}(t) \in-\partial \Phi_{I I}^{*,-}\left(\lambda_{I I}(t)\right)
\end{array}\right. \\
& \text { 企 } \\
& x^{\prime}(t)-B^{I I}\left(D_{I I}+\partial \Phi_{I I}^{*,-}\right)^{-1}\left(-C_{I I} x(t)\right)-A x(t)-f(t) \in-B^{I} \partial \Phi_{I}\left(C_{I} x(t)\right) \\
& \text { 企 } \\
& V x^{\prime}(t)-V B^{I I}\left(D_{I I}+\partial \Phi_{I I}^{*,-}\right)^{-1}\left(-C_{I I} V^{-1} V x(t)\right)-V A V^{-1} V x(t)-V f(t) \in-V^{-1} V^{2} B^{I} \partial \Phi_{I}\left(C_{I} V^{-1} V x(t)\right) \\
& \text { 企 } \\
& X^{\prime}(t)-V B^{I I}\left(D_{I I}+\partial \Phi_{I I}^{*,-}\right)^{-1}\left(-C_{I I} V^{-1} X(t)\right)-V A V^{-1} X(t)-V f(t) \in-V^{-1} C_{I}^{T} \partial \Phi_{I}\left(C_{I} V^{-1} X(t)\right) \\
& \text { 企 } \\
& X^{\prime}(t)-V A V^{-1} X(t)-V B^{I I}\left(D_{I I}+\partial \Phi_{I I}^{*,-}\right)^{-1}\left(-C_{I I} V^{-1} X(t)\right)-V f(t) \in-\partial \Xi_{I}(X(t))
\end{aligned}
$$

from which one deduces (17). The case $p_{I}=0$ (resp. $p_{I I}=0$ ) can be deduced from the previous relations in removing the terms indexed by $I$ (resp. II). The system has therefore been transformed from (a) to (b) in Fig. 1, which are equivalent representations. As will be made clear in the next section, the transformation consists of inserting the Lipschitz continuous part of the multivalued nonlinearity, into the continuous dynamics of the system. 


\subsubsection{The operator $x \mapsto V B^{I I}\left(D_{I I}+\partial \Phi_{I I}^{*,-}\right)^{-1}\left(-C_{I I} V^{-1} x\right)$}

In this section, we suppose that $p_{I I} \geq 1$. The following two results give conditions on $D_{I I}$ and $\Phi_{I I}(\cdot)$ ensuring that the operator $z \in \mathbb{R}^{p_{I I}} \mapsto\left(D_{I I}+\partial \Phi_{I I}^{*,-}\right)^{-1}(z)$ is well-defined, single-valued and Lipschitz continuous, i.e. such that Assumption 4 is satisfied. The operator $\Lambda_{I I}: x \in \mathbb{R}^{n} \mapsto V B^{I I}\left(D_{I I}+\partial \Phi_{I I}^{*,-}\right)^{-1}\left(-C_{I I} V^{-1} x\right)$ is then consequently also well-defined, singlevalued and Lipschitz continuous.

Proposition 1. Suppose that $D_{I I}$ is positive definite and $\Phi_{I I}(\cdot)$ is proper convex and lower semicontinuous. Then the operator $\left(D_{I I}+\partial \Phi_{I I}^{*,-}\right)^{-1}$ is well-defined, single-valued and Lipschitz continuous.

Proof. The conditions of the proposition ensure (see e.g. [22]) that for all $q \in \mathbb{R}^{p_{\text {II }}}$ there exists a unique $z=z(q) \in \mathbb{R}_{+}^{p_{I I}}$ such that

$$
\left\langle D_{I I} z-q, v-z\right\rangle+\Phi_{I I}^{*,-}(v)-\Phi_{I I}^{*,-}(z) \geq 0, \quad \forall v \in \mathbb{R}^{p_{I I}},
$$

that is equivalent to $D_{I I} z-q \in-\partial \Phi_{I I}^{*,-}(z)$. The operator $\left(D_{I I}+\partial \Phi_{I I}^{*,-}\right)^{-1}: \mathbb{R}^{p} \rightarrow \mathbb{R}^{p} ; q \mapsto\left(D_{I I}+\partial \Phi_{I I}^{*,-}\right)^{-1}(q)$ is thus welldefined and single-valued. It is also Lipschitz continuous. Indeed, let $q_{1}, q_{2} \in \mathbb{R}^{p}$ be given. Set $z_{1}=\left(D_{I I}+\partial \Phi_{I I}^{*,-}\right)^{-1}\left(q_{1}\right)$ and $z_{2}=\left(D_{I I}+\partial \Phi_{I I}^{*,-}\right)^{-1}\left(q_{2}\right)$. We have $\left\langle D_{I I} z_{1}-q_{1}, z_{2}-z_{1}\right\rangle+\Phi_{I I}^{*,-}\left(z_{2}\right)-\Phi_{I I}^{*,-}\left(z_{1}\right) \geq 0$ and $\left\langle D_{I I} z_{2}-q_{2}, z_{1}-z_{2}\right\rangle+$ $\Phi_{I I}^{*,-}\left(z_{1}\right)-\Phi_{I I}^{*,-}\left(z_{2}\right) \geq 0$ and thus $\left\langle D_{I I}\left(z_{1}-z_{2}\right), z_{1}-z_{2}\right\rangle \leq\left\langle q_{1}-q_{2}, z_{1}-z_{2}\right\rangle$. The matrix $D$ is positive definite and thus $\left(\forall x \in \mathbb{R}^{n}\right):\left\langle D_{I I} x, x\right\rangle \geq \frac{\lambda_{1}\left(D_{I I}+D_{I I}^{T}\right)}{2}\|x\|^{2}$, where $\lambda_{1}\left(D_{I I}+D_{I I}^{T}\right)>0$ is the smallest eigenvalue of the matrix $D_{I I}+D_{I I}^{T}$. Thus $\left\|z_{1}-z_{2}\right\| \leq \frac{2}{\lambda_{1}\left(D_{I I}+D_{I I}^{T}\right)}\left\|q_{1}-q_{2}\right\|$ and then:

$$
\left\|\left(D_{I I}+\partial \Phi_{I I}^{*,-}\right)^{-1}\left(q_{1}\right)-\left(D+\partial \Phi_{I I}^{*,-}\right)^{-1}\left(q_{2}\right)\right\| \leq \frac{2}{\lambda_{1}\left(D_{I I}+D_{I I}^{T}\right)}\left\|q_{1}-q_{2}\right\| .
$$

Let us recall that a $P$-matrix is a matrix with all its principal minors positive [23].

Proposition 2. Suppose that $D_{I I}$ is a P-matrix and $\Phi_{I I}(\cdot)=\Psi_{\mathbb{R}_{+}^{p_{I}}}(\cdot)$. Then the operator $\left(D_{I I}+\partial \Phi_{I I}^{*,-}\right)^{-1}$ is well-defined, singlevalued and Lipschitz continuous.

Proof. Here $\left(\forall z \in \mathbb{R}_{I I}^{p}\right): \quad \Phi_{I I}^{*,-}(z)=\Psi_{\mathbb{R}_{+}^{p_{I}}}^{*}(-z)=\Psi_{\mathbb{R}_{-}^{p_{I I}}}(-z)=\Psi_{\mathbb{R}_{+}^{p_{I I}}}(z)$. Thus $\left(D_{I I}+\partial \Phi_{I I}^{*,-}\right)^{-1} \equiv\left(D_{I I}+\partial \Psi_{\mathbb{R}_{+}^{p_{I I}}}\right)^{-1}$. It is easy to check that $z=\left(D_{I I}+\partial \Psi_{\mathbb{R}_{+}^{p_{I I}}}\right)^{-1}(q)$ if and only if $z$ is solution of the complementarity problem: $z \in \mathbb{R}_{+}^{p_{I I}}, D_{I I} z-q \in$ $\mathbb{R}_{+}^{p_{I I}},\left\langle z, D_{I I} z-q\right\rangle=0$. The matrix $D_{I I}$ is assumed to be a $P$-matrix and the well-defined single-valued Lipschitz continuity property of the solution map of the complementarity problem is in this case a well-known result, see e.g. [23].

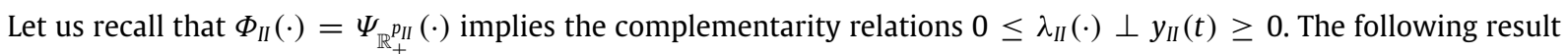
concerns the important class of positive semidefinite matrices.

Proposition 3. Suppose that $D_{\text {II }}$ is positive semidefinite, i.e.

$$
\forall z \in \mathbb{R}^{p_{I I}}:\left\langle D_{I I} z, z\right\rangle \geq 0 .
$$

Suppose also that

$$
\begin{aligned}
& \operatorname{dom}\left(\Phi_{I I}^{*,-}\right)=\overline{\operatorname{dom}\left(\Phi_{I I}^{*,-}\right)} \\
& \left(\operatorname{dom}\left(\Phi_{I I}^{*,-}\right)\right)_{\infty} \cap \operatorname{ker}\left(D_{I I}+D_{I I}^{T}\right) \cap\left\{z \in \mathbb{R}^{p_{I I}}: D_{I I} z \in\left(\operatorname{dom}\left(\left(\Phi_{I I}^{*,-}\right)_{\infty}\right)\right)^{\star}\right\}=\{0\}
\end{aligned}
$$

and

$$
\mathcal{R}\left(C_{I I}\right) \subset \mathcal{R}\left(D_{I I}+D_{I I}^{T}\right) \subset \operatorname{ker}\left(B^{I I}\right) .
$$

Then the operator $x \mapsto V B^{I I}\left(D_{I I}+\partial \Phi_{I I}^{*,-}\right)^{-1}\left(-C_{I I} V^{-1} x\right)$ is well-defined, single-valued and Lipschitz continuous.

Proof. The existence for any $q \in \mathbb{R}_{+}^{p_{\text {II }}}$ of at least one solution of the variational inequality

$$
z=z(q) \in \mathbb{R}_{+}^{p_{I I}}:\left\langle D_{I I} z-q, v-z\right\rangle+\Phi_{I I}^{*,-}(v)-\Phi_{I I}^{*,-}(z) \geq 0, \quad \forall v \in \mathbb{R}^{p_{I I}}
$$

follows from assumptions (18), (19), (20) and Corollary 3.6 in [22]. Let $x_{1}, x_{2} \in \mathbb{R}^{p_{I I}}$ and set $q_{1}=-C_{I I} V^{-1} x_{1}, q_{2}=-C_{I I} V^{-1} x_{2}$, $z_{1}=z\left(q_{1}\right)$ and $z_{2}=z\left(q_{2}\right)$. We have

$$
\left\langle D_{I I} z_{1}-q_{1}, v-z_{1}\right\rangle+\Phi_{I I}^{*,-}(v)-\Phi_{I I}^{*,-}\left(z_{1}\right) \geq 0, \quad \forall v \in \mathbb{R}^{p I I}
$$

and

$$
\left\langle D_{I I} z_{2}-q_{2}, v-z_{2}\right\rangle+\Phi_{I I}^{*,-}(v)-\Phi_{I I}^{*,-}\left(z_{2}\right) \geq 0, \quad \forall v \in \mathbb{R}^{p_{I I}}
$$


from which we deduce that

$$
\left\langle D_{I I}\left(z_{2}-z_{1}\right), z_{2}-z_{1}\right\rangle \leq\left\langle q_{2}-q_{1}, z_{2}-z_{1}\right\rangle .
$$

Let $H=\operatorname{ker}\left(D_{I I}+D_{I I}^{T}\right)$. We denote by $P_{H}$ the orthogonal projector from $\mathbb{R}^{p_{I I}}$ onto $H$ and by $P_{H^{\perp}}$ the orthogonal projector from $\mathbb{R}^{p_{I I}}$ onto the orthogonal space $H^{\perp}=\mathcal{R}\left(D_{I I}+D_{I I}^{T}\right)$. There exists a constant $c>0$ such that

$$
\forall z \in \mathbb{R}^{p_{I I}}:\left\langle D_{I I} z, z\right\rangle \geq c\left\|P_{H^{\perp}} z\right\|^{2} .
$$

We know from (21) that $q_{i}=-C_{I I} V^{-1} x_{1} \in \mathcal{R}\left(C_{I I}\right) \subset H^{\perp}=\mathcal{R}\left(D_{I I}+D_{I I}^{T}\right)$ so that $P_{H}\left(q_{i}\right)=0$ and $P_{H^{\perp}}\left(q_{i}\right)=q_{i}(i=1,2)$. Thus

$$
\begin{aligned}
\left\langle q_{2}-q_{1}, z_{2}-z_{1}\right\rangle & =\left\langle P_{H}\left(q_{2}-q_{1}\right), P_{H}\left(z_{2}-z_{1}\right)\right\rangle+\left\langle P_{H^{\perp}}\left(q_{2}-q_{1}\right), P_{H^{\perp}}\left(z_{2}-z_{1}\right)\right\rangle \\
& =\left\langle q_{2}-q_{1}, P_{H^{\perp}}\left(z_{2}-z_{1}\right)\right\rangle \leq\left\|q_{2}-q_{1}\right\|\left\|P_{H^{\perp}}\left(z_{2}-z_{1}\right)\right\| \\
& \leq\left\|C_{I I} V^{-1}\right\|\left\|x_{2}-x_{1}\right\|\left\|P_{H^{\perp}}\left(z_{2}-z_{1}\right)\right\| .
\end{aligned}
$$

It results from (22), (23) and (24) that

$$
\left\|P_{H^{\perp}}\left(z_{2}-z_{1}\right)\right\| \leq \frac{1}{c}\left\|C_{I I} V^{-1}\right\|\left\|x_{2}-x_{1}\right\| .
$$

Then recalling that by assumption $H^{\perp} \subset \operatorname{ker}\left(B^{I I}\right)$, we get

$$
\begin{aligned}
\left\|V B^{I I}\left(z_{2}-z_{1}\right)\right\| & =\left\|V B^{I I}\left(P_{H^{\perp}}\left(z_{2}-z_{1}\right)\right)+V B^{I I}\left(P_{H}\left(z_{2}-z_{1}\right)\right)\right\|=\left\|V B^{I I}\left(P_{H^{\perp}}\left(z_{2}-z_{1}\right)\right)\right\| \\
& \leq \frac{1}{c}\left\|V B^{I I}\right\|\left\|C_{I I} V^{-1}\right\|\left\|x_{2}-x_{1}\right\| .
\end{aligned}
$$

This means that the operator $x \mapsto V B^{I I}\left(D_{I I}+\partial \Phi_{I I}^{*,-}\right)^{-1}\left(-C_{I I} V^{-1} x\right)$ is well-defined, single-valued and Lipschitz continuous.

We now may state the next result as a corollary of Proposition 3.

Corollary 1. Let $D_{\text {II }}$ be positive semidefinite, and suppose that $\Phi_{I I}(\cdot)=\Psi_{K}(\cdot)$ for some closed convex cone $K \subset \mathbb{R}^{p_{I I}}$. Then if $K^{0} \cap \operatorname{ker}\left(D_{I I}+D_{I I}^{T}\right)=\{0\}$ and $\mathcal{R}\left(C_{I I}\right) \subset \mathcal{R}\left(D_{I I}+D_{I I}^{T}\right) \subset \operatorname{ker}\left(B^{I I}\right)$, the operator $x \mapsto V B^{I I}\left(D_{I I}+\partial \Phi_{I I}^{*,-}\right)^{-1}\left(-C_{I I} V^{-1} x\right)$ is well-defined, single-valued and Lipschitz continuous.

The proof relies on the fact that $\operatorname{dom}\left(\Psi_{K}\right)=K$ and $\Psi_{K}^{*}(\cdot)=\Psi_{K^{0}}(\cdot)$. Notice that in such a case one has the cone complementarity problem $K \ni y_{I I}(t) \perp \lambda_{I I}(t) \in K^{*}=-K^{0}$. If $D_{I I}$ is definite positive then Proposition 1 applies.

\subsubsection{Existence and uniqueness of solutions}

Let us now state the well-posedness result relying on Kato's Theorem.

Theorem 1. Suppose that Assumptions 1-4 hold. Then for any $X_{0} \in \operatorname{Dom}\left(\partial \Xi_{I}\right)$ there exists a unique $X \in C^{0}\left(\mathbb{R}_{+} ; \mathbb{R}^{n}\right)$ such that $X^{\prime} \in L_{\mathrm{loc}}^{\infty}\left(\mathbb{R}_{+} ; \mathbb{R}^{n}\right), X(\cdot)$ right-differentiable on $\mathbb{R}_{+}$and:

$$
\begin{aligned}
& X(t) \in \operatorname{Dom}\left(\partial \Xi_{I}\right), \quad(\forall t \geq 0), \\
& \left.\left\langle X^{\prime}(t)-V A V^{-1} X(t)-\Lambda_{I I}(X(t))-V f(t), v-X(t)\right\rangle+\Xi_{I}(v)-\Xi_{I}(X(t)) \geq 0, \quad \forall v \in \mathbb{R}^{n}, \text { (a.e. } t \geq 0\right), \\
& X(0)=X_{0} .
\end{aligned}
$$

Proof. The mapping $X \mapsto-V A V^{-1} X-\Lambda_{I I}(X)$ is Lipschitz continuous. We may then apply a version of Kato's Theorem (see Corollary 2.2 in [24]) to guarantee the existence and the uniqueness of the solution $X(\cdot)$ of problem (25)-(27).

Then for $x_{0} \in V^{-1} \operatorname{Dom}\left(\Xi_{I}\right)$, the solution $x(\cdot)$ of the problem $\operatorname{NSDS}\left(A, B, C, D, \varphi_{1}, \ldots, \varphi_{p}, f, x_{0}\right)$ is uniquely defined by the formula:

$$
x(t)=V^{-1} X(t) .
$$

It results that $\lambda_{I I}$ (case $p_{I I} \geq 1$ ) is uniquely defined by the formula

$$
\lambda_{I I}(t)=\left(D_{I I}+\partial \Phi_{I I}^{*,-}\right)^{-1}\left(-C V^{-1} X(t)\right) .
$$

The uniqueness of $y$ is then a consequence of the formula:

$$
y(t)=C V^{-1} X(t)+D \lambda(t)=C V^{-1} X(t)+D_{I I} \lambda_{I I}(t) .
$$

The uniqueness of $\lambda_{I}$ (case $p_{I} \geq 1$ ) is not in general guaranteed. However, if $\lambda_{I}^{1}$ and $\lambda_{I}^{2}$ denote two solutions then necessarily

$$
(\forall t \geq 0): B^{I} \lambda_{I}^{1}(t)=B^{I} \lambda_{I}^{2}(t) .
$$

It results that if $\operatorname{rank}\left\{B^{I}\right\}=p_{I}$ then the uniqueness of $\lambda_{I}$ is also ensured. 
Remark 2. Let $X=V x$ be given. We note that if $p_{I} \geq 1$ then $X \in \operatorname{Dom}\left(\partial \Xi_{I}\right) \Leftrightarrow C_{I} V^{-1} X \in \operatorname{Dom}\left(\partial \Phi_{I}\right) \Leftrightarrow C_{I} x \in \operatorname{Dom}\left(\partial \Phi_{I}\right)$. If $p_{I}=0$ then $X \in \operatorname{Dom}\left(\partial \Xi_{I}\right) \Leftrightarrow X \in \mathbb{R}^{n} \Leftrightarrow x \in \mathbb{R}^{n}$.

The case $p_{I}=p$ is given in the following corollary. Here $\Lambda \equiv 0$ and we may set

$\left(\forall w \in \mathbb{R}^{n}\right): \Xi_{I}(w)=\Xi(w):=\Phi\left(C V^{-1} w\right)$.

Assumption 4 is here useless and we obtain the result:

Corollary 2. Let $p_{I}=p$. Suppose that Assumptions 1-3 hold. Then for any $X_{0} \in \operatorname{dom}(\Xi)$ there exists a unique $X \in C^{0}\left(\mathbb{R}_{+} ; \mathbb{R}^{n}\right)$ such that $X^{\prime} \in L_{\text {loc }}^{\infty}\left(\mathbb{R}_{+} ; \mathbb{R}^{n}\right), X(\cdot)$ right-differentiable on $\mathbb{R}_{+}$and:

$$
\begin{aligned}
& X(t) \in \operatorname{Dom}(\partial \Xi), \quad(\forall t \geq 0), \\
& \left.\left\langle X^{\prime}(t)-V A V^{-1} X(t)-V f(t), v-X(t)\right\rangle+\Xi(v)-\Xi(X(t)) \geq 0, \quad \forall v \in \mathbb{R}^{n}, \text { (a.e. } t \geq 0\right), \\
& X(0)=X_{0} . \quad \square
\end{aligned}
$$

Under the conditions of the Corollary it follows that for $x_{0} \in \mathbb{R}^{n}$ such that $C x_{0} \in \operatorname{Dom}(\partial \Phi)$, the function

$$
x(\cdot)=V^{-1} X(\cdot)
$$

is the unique solution of the problem:

$$
\left\{\begin{array}{l}
x^{\prime}(t)=A x(t)+B \lambda(t)+f(t) \quad(\text { a.e. } t \geq 0) \\
x(0)=x_{0} \\
y(t)=C x(t) \quad(\forall t \geq 0) \\
\lambda(t) \in-\partial \Phi(y(t)) \quad(\forall t \geq 0)
\end{array}\right.
$$

Remark 3. The function $y$ is uniquely defined by the formula $y=C x$ and if $\operatorname{rank}(B)=p$ then $\lambda$ is also uniquely defined by the formula $B \lambda=x^{\prime}-A x-f$.

Let us now state the case $p_{I I}=p$. Then $\Xi_{I} \equiv 0$. Here Assumptions 2 and 3 are useless and we obtain the following result:

Corollary 3. Let $p_{I I}=p$. Suppose that Assumptions 1 and 4 hold. Then for any $X_{0} \in \mathbb{R}^{n}$ there exists a unique $X \in C^{0}\left(\mathbb{R}_{+} ; \mathbb{R}^{n}\right)$ such that $X^{\prime} \in L_{\mathrm{loc}}^{\infty}\left(\mathbb{R}_{+} ; \mathbb{R}^{n}\right)$ and $X(\cdot)$ right-differentiable on $\mathbb{R}_{+}$such that:

$$
\begin{aligned}
& \left.X^{\prime}(t)-A X(t)-B\left(D+\partial \Phi^{*,-}\right)^{-1}(-C X(t))-f(t)=0 \quad \text { (a.e. } t \geq 0\right), \\
& X(0)=X_{0} .
\end{aligned}
$$

Then for $X_{0} \in \mathbb{R}^{n}$ the function $X(\cdot)$ is the unique solution of the problem:

$$
\left\{\begin{array}{l}
X^{\prime}(t)=A X(t)+B \lambda(t)+f(t) \quad(\text { a.e. } t \geq 0) \\
X(0)=X_{0} \\
y(t)=C X(t)+D \lambda(t) \quad(\forall t \geq 0) \\
\lambda(t) \in-\partial \Phi(y(t)) \quad(\forall t \geq 0)
\end{array}\right.
$$

Remark 4. If $\operatorname{rank}(B)=p$ then $\lambda$ is uniquely defined by the formula $B \lambda=X^{\prime}-A X-f$ and $y$ is then also uniquely defined by the formula $y=C X+D \lambda$.

Remark 5. If the operator $\left(D+\partial \Phi^{*,-}\right)^{-1}$ is single-valued (see Propositions 1 and 2 ) then $\lambda$ is uniquely defined by the formula $\lambda=\left(D+\partial \Phi^{*,-}\right)^{-1}(-C X)$. Then $y$ is also uniquely defined by the formula $y=C X+D \lambda$.

\subsubsection{A physical example}

Let us consider the electrical system of Fig. 2 that is composed of two resistors $R$ with voltage/current law $u(t)=R i(t)$, four capacitors $C$ with voltage/current law $C u^{\prime}(t)=i(t)$, and two ideal diodes with characteristics $0 \leq v_{1}(t) \perp i_{1}(t) \geq 0$ and $0 \leq v_{2}(t) \perp i_{3}(t) \geq 0$ respectively. The state variables are $x_{1}(t)=\int_{0}^{t} i_{1}(t) \mathrm{d} t, x_{2}(t)=\int_{0}^{t} i_{2}(t) \mathrm{d} t, x_{3}(t)=v_{2}(t)$, and $\lambda_{1}(t)=-i_{3}(t), \lambda_{2}(t)=v_{1}(t)$.

The dynamics of this circuit is given by:

$$
\begin{aligned}
& \left(\begin{array}{l}
x_{1}^{\prime}(t) \\
x_{2}^{\prime}(t) \\
x_{3}^{\prime}(t)
\end{array}\right)=\left(\begin{array}{ccc}
\frac{-2}{R C} & \frac{1}{C} & 0 \\
\frac{1}{C} & \frac{-2}{R C} & 1 \\
0 & 0 & 0
\end{array}\right)\left(\begin{array}{l}
x_{1}(t) \\
x_{2}(t) \\
x_{3}(t)
\end{array}\right)+\left(\begin{array}{cc}
0 & \frac{1}{R} \\
0 & 0 \\
\frac{1}{C} & 0
\end{array}\right) \lambda(t), \\
& 0 \leq \lambda(t) \perp y(t)=\left(\begin{array}{ccc}
0 & 0 & 1 \\
\frac{-2}{R C} & \frac{1}{R C} & 0
\end{array}\right)\left(\begin{array}{l}
x_{1}(t) \\
x_{2}(t) \\
x_{3}(t)
\end{array}\right)+\left(\begin{array}{ll}
0 & 0 \\
0 & \frac{1}{R}
\end{array}\right) \lambda(t) \geq 0 .
\end{aligned}
$$




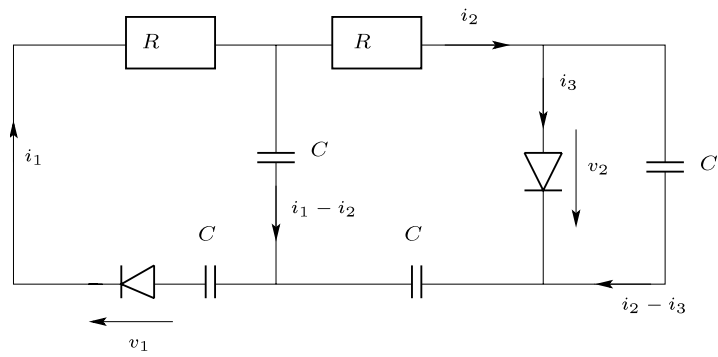

Fig. 2. Electrical circuit with capacitors, resistors and ideal diodes.

The matrices $A, B, C$ and $D$ are easily identified. Assumptions 1-4 are satisfied as one may check that Proposition 1 (or Proposition 2) and consequently Theorem 1 apply. One has $\varphi_{I I}(\cdot)=\psi_{K}(\cdot)$ with $K=\mathbb{R}^{+}$. It is noteworthy that one may consider any other electronic devices with current/voltage laws $v_{1} \in \partial \varphi_{2}\left(i_{1}\right)$ and $-i_{3} \in \partial \varphi_{1}\left(v_{2}\right)$ provided the functions $\varphi_{1}(\cdot)$ and $\varphi_{2}(\cdot)$ are proper, convex lower semicontinuous. Doing so our framework encompasses linear complementarity systems and allows for a much larger set of nonsmooth nonlinear characteristics of the electronic devices, see [25] for some examples.

\subsection{Well-posedness by maximal monotonicity}

It is noteworthy that the application of condition (21) in Proposition 3 implies that the symmetric part of $D$ has a large enough range. This is not the case when $D$ is skew-symmetric (see a four-diode bridge full wave rectifier in Example 4 in [22]) or when its symmetric part has an orthogonal range to the range of $C$ (see a four-diode bridge wave rectifier in Section 14.2.1 in [26], despite the condition (20) may be satisfied). This motivates us to look for another path to show the well-posedness of $(2)$.

\subsubsection{The existence and uniqueness result}

In this section we shall not make the assumption that $D$ possesses a structure as in (6), however a stronger assumption than Assumption 2 is made:

Assumption 5. (i) There exists a matrix $P=P^{T}>0$ such that $P B=C^{T}$, and (ii) $D$ is positive semidefinite.

Using the same notations as in Section 3.1, one has $\lambda \in-\partial \Phi(C x+D \lambda)$, and $C x+D \lambda \in-\partial \Phi^{*,-}(\lambda)$. Let us define the operator $\lambda \mapsto D \lambda+\partial \Phi^{*,-}(\lambda)$. In view of the assumptions on $\Phi(\cdot)$ and of Assumption 5 , the operator $\left(D \cdot+\partial \Phi^{*,-}(\cdot)\right)$ is maximal monotone, being the sum of two maximal monotone operators with $\operatorname{dom}(D \cdot)=\mathbb{R}^{p}[21$, Corollary 12.44]. Now from [21, Exercise 12.8] it follows that the inverse operator $\left(D \cdot+\partial \Phi^{*,-}\right)^{-1}(\cdot)$ is maximal monotone as well. It is now easy to see that the Lur'e system in (2) may be rewritten equivalently as:

$$
x^{\prime}(t) \in A x(t)+B\left(D \cdot+\partial \Phi^{*,-}\right)^{-1}(-C x(t))+f(t) .
$$

Let $R$ be the symmetric positive definite square root of $P$, i.e. $R^{2}=P$. Let us perform the state variable transformation $z=-R x$. Using Assumption 5 the system in (34) may be rewritten as:

$$
z^{\prime}(t) \in R A R^{-1} z(t)-R^{-1} C^{T}\left(D \cdot+\partial \Phi^{*,-}\right)^{-1}\left(C R^{-1} z(t)\right)-R f(t) .
$$

Since $R$ is symmetric it now follows from [21, Theorem 12.43] that the operator $z \mapsto R^{-1} C^{T}\left(D \cdot+\partial \Phi^{*,-}\right)^{-1}\left(C R^{-1} z\right)$ is maximal monotone, provided $\mathcal{R}\left(C R^{-1}\right) \cap \operatorname{rint}\left(\operatorname{Dom}\left(\left(D \cdot+\partial \Phi^{*,-}\right)^{-1}\right)\right) \neq \emptyset$.

We now may state the following:

Theorem 2. Let Assumptions 1 and 5 hold, and suppose that $\mathcal{R}\left(C R^{-1}\right) \cap \operatorname{rint}\left(\operatorname{Dom}\left(\left(D \cdot+\partial \Phi^{*,-}\right)^{-1}\right)\right) \neq \emptyset$. Let also $x_{0} \in \mathbb{R}^{n}$ such that $-C x_{0} \in \operatorname{Dom}\left(\left(D \cdot+\partial \Phi^{*,-}\right)^{-1}\right)\left(=\mathcal{R}\left(D \cdot+\partial \Phi^{*,-}\right)\right)$. Then the Lur'e system in (2) possesses a unique solution that is Lipschitz continuous.

Proof. The proof follows directly from the above developments and the application of [27, Proposition 3] (see also [28, Proposition 4.3]).

It is noteworthy that since $\mathcal{R}\left(D \cdot+\partial \Phi^{*,-}\right)=\operatorname{Dom}\left(\left(D \cdot+\partial \Phi^{*,-}\right)^{-1}\right)$ then the condition of the theorem is equivalent to $\mathcal{R}\left(C R^{-1}\right) \cap \operatorname{rint}\left(\mathcal{R}\left(D \cdot+\partial \Phi^{*,-}\right)\right) \neq \varnothing$. It is also noteworthy that contrary to Section 3.1 where the operator $x \mapsto$ $V B^{I I}\left(D_{I I}+\partial \Phi_{I I}^{*,-}\right)^{-1}\left(-C_{I I} V^{-1} x\right)$ is supposed (or shown) to be single-valued, here we allow for multivaluedness. Notice that there is no obstacle to consider "outputs" $y=C x+D \lambda+E$ with constant $E$ of appropriate dimension. Indeed this does not preclude for the above reasoning to work because considering composition with affine mappings preserves also the maximal monotonicity [21, Theorem 12.43], provided the range intersection condition is still satisfied. 


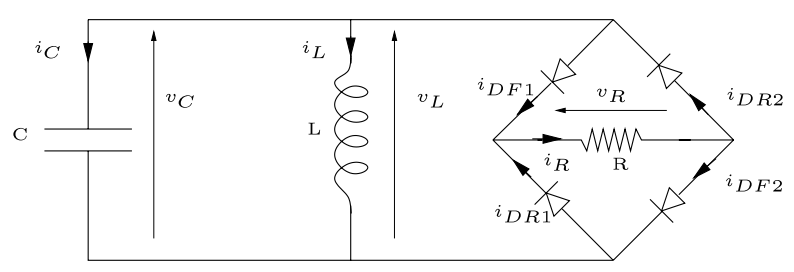

Fig. 3. A 4-diode bridge wave rectifier.

Notice that if $p_{I}=0$ and if we rely on the results of Section 3.1, then we impose that the operator $\left(D \cdot+\partial \Phi^{*,-}\right)^{-1}$ is single-valued, which is not the case in this section where it is allowed to be multivalued.

Remark 6. Assumption 5(i) implies that the so-called Markov parameter $C B=B^{T} P B \geq 0$ and is symmetric. This is a relative degree condition on the quadruplet $(A, B, C, D)$, in the sense that if $D=0$ and $p=1$ then $C B>0$ and the relative degree is equal to one.

\subsubsection{A physical example}

Let us consider the four-diode bridge wave rectifier in Fig. 3, with a capacitor $\mathbf{C}>$, an inductance $\mathbf{L}>0$, a resistor $\mathbf{R}>0$. Its dynamics is given by [26]:

$$
\begin{aligned}
& {\left[\begin{array}{l}
x_{1}^{\prime}(t) \\
x_{2}^{\prime}(t)
\end{array}\right]=\left[\begin{array}{cc}
0 & -\frac{1}{\mathbf{C}} \\
\frac{1}{\mathbf{L}} & 0
\end{array}\right]\left[\begin{array}{l}
x_{1}(t) \\
x_{2}(t)
\end{array}\right]+\left[\begin{array}{cccc}
0 & 0 & -\frac{1}{\mathbf{C}} & \frac{1}{\mathbf{C}} \\
0 & 0 & 0 & 0
\end{array}\right] \lambda(t)} \\
& 0 \leq y(t) \perp \lambda(t) \geq 0
\end{aligned}
$$

where $x_{1}=v_{L}, x_{2}=i_{L}, \lambda=\left(-v_{D R 1}-v_{D F 2} i_{D F 1} i_{D R 2}\right)^{T}, y=\left(i_{D R 1} i_{D F 2}-v_{D F 1}-v_{D R 2}\right)^{T}$ and

$$
y=\left[\begin{array}{cc}
0 & 0 \\
0 & 0 \\
-1 & 0 \\
1 & 0
\end{array}\right]\left[\begin{array}{l}
x_{1} \\
x_{2}
\end{array}\right]+\left[\begin{array}{cccc}
\frac{1}{\mathbf{R}} & \frac{1}{\mathbf{R}} & -1 & 0 \\
\frac{1}{\mathbf{R}} & \frac{1}{\mathbf{R}} & 0 & -1 \\
1 & 0 & 0 & 0 \\
0 & 1 & 0 & 0
\end{array}\right] \lambda .
$$

Notice that in this example one has $n=2$ and $p=4$. The matrix $D$ is full rank, semi-definite positive. The relation $P B=C^{T}$ holds with $P=\left(\begin{array}{cc}\mathbf{c} & 0 \\ 0 & p_{22}\end{array}\right), p_{22}>0$, where $\mathbf{C}>0$ is the capacitor parameter. One may check that condition (20) is satisfied, however (21) is not.

As a second example, let us consider another diode-bridge that is taken from [22, Example 4]. It is simply obtained from the circuit of Fig. 3 by dropping the capacitor and the inductance outside the bridge, and adding a capacitor $\mathbf{C}$ in parallel with the resistor inside the bridge. The state $x$ is the voltage across the capacitor. We assume that each diode has a current/voltage law of the form $V_{k} \in-\partial \varphi_{k}\left(i_{k}\right), k=1,2,3,4$, for some convex, proper lower semicontinuous functions $\varphi_{k}(\cdot)$. The dynamics of this circuit is given by:

$$
\begin{aligned}
& x^{\prime}(t)=-\frac{1}{\mathbf{R C}} x(t)+\left(\frac{1}{\mathbf{C}} 0 \frac{1}{\mathbf{C}} 0\right) \lambda(t) \\
& y(t)=\left(\begin{array}{l}
1 \\
0 \\
1 \\
0
\end{array}\right) x(t)+\left(\begin{array}{cccc}
0 & -1 & 0 & 0 \\
1 & 0 & 1 & -1 \\
0 & -1 & 0 & 0 \\
0 & 1 & 0 & 0
\end{array}\right) \lambda(t)
\end{aligned}
$$

with $y_{1}=V_{D R 1}, y_{2}=i_{D F 2}, y_{3}=V_{D F 1}, y_{4}=V_{D R 2}$, and $\lambda=\left(i_{D R 1} V_{D F 2} i_{D F 1} i_{D R 2}\right)^{T}$. The matrix $D$ has rank 2 , it is positive semidefinite since it is skew symmetric. Assumption 5 is satisfied with $P=\mathbf{C}>0$. We may then choose $R=\sqrt{\mathbf{C}}$. One has

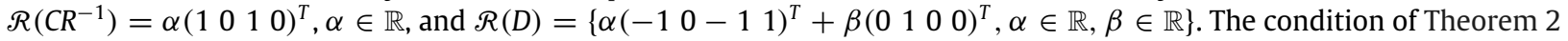
is satisfied for a large range of functions $\varphi_{i}(\cdot)$. This is the case in the complementarity framework if $\varphi_{i}(\cdot)=\psi_{K_{i}}(\cdot)$ with $K_{i}=\mathbb{R}^{+}$: then $\varphi_{i}^{*}(\cdot)=\psi_{K_{i}^{\circ}}(\cdot)$ where $K_{i}^{\circ}=\mathbb{R}^{-}$is the polar cone to $K_{i}$.

Remark 7 (Time-discretization). The implicit Euler time-stepping method studied in [28] can be used to discretize the Lur'e system under study. Under the stated assumptions the method may be shown to converge, and to be of order $\frac{1}{2}$ or 1 (in 
the case where $\varphi_{i}(\cdot)$ are the indicator functions of closed convex sets, i.e. the complementarity framework). Therefore our results permit one to encompass the results of [19], since we do not assume the stability of the matrix $A$ and we may have $p>n$. Moreover we do not need that the matrix $\left(\begin{array}{c}B \\ D+D^{T}\end{array}\right)$ be full column rank as in $[19,20]$. It is noteworthy that for such types of nonsmooth dynamical systems, event-driven methods [26] may fail to integrate the system even on arbitrarily small intervals of time, and time-stepping methods are the only available methods.

\subsubsection{State observer design}

This example illustrates how the foregoing results on well-posedness may be used for the design of asymptotically stable state observers for the Lur'e system in (2). The state $x$ and the multiplier $\lambda$ are not available for feedback. The measured output of (2) is supposed to possess the general form: $z=G x+F \lambda, z \in \mathbb{R}^{m}, G \in \mathbb{R}^{m \times n}, F \in \mathbb{R}^{m \times p}$. It may also be assumed that $m<n$. The following state observer is proposed:

$$
\left\{\begin{array}{l}
\xi^{\prime}(t)=A \xi(t)+B \gamma(t)+f(t)+L(z(t)-G \xi(t)-F \gamma(t)) \\
\gamma(t) \in-\partial \Phi(C \xi(t)+D \gamma(t))
\end{array}\right.
$$

which is equivalently rewritten as:

$$
\left\{\begin{array}{l}
\xi^{\prime}(t)=(A-L G) \xi(t)+(B-L F) \gamma(t)+L G x(t)+L F \lambda(t)+f(t) \\
\gamma(t) \in-\partial \Phi(C \xi(t)+D \gamma(t))
\end{array}\right.
$$

Let us make the following assumption:

Assumption 6. The observed dynamics in (2) is well-posed, i.e. it possesses for each admissible initial condition a unique right-differentiable continuous solution with bounded derivative, defined on $[0,+\infty)$.

Starting from this assumption, we may consider the term $L G x(t)+L F \lambda(t)+f(t)$ as an exogenous function of time for the observer dynamics, denoted as $g(t)$. The observer dynamics in (40) is therefore under the general form in (2). Its wellposedness may be analyzed similarly. Notice that we may equivalently rewrite (40) as:

$$
\left\{\begin{array}{l}
\xi^{\prime}(t)=(A-L G) \xi(t)+(B-L F) \gamma(t)+L G x(t)+L F \lambda(t)+f(t) \\
C \xi(t)+D \gamma(t) \in-\partial \Phi^{*,-}(\gamma(t))
\end{array}\right.
$$

Let us suppose that both the observed system and the observer are well-posed, and let us form the so-called error dynamics with state vector $e=x-\xi$ :

$$
\left\{\begin{array}{l}
\mathrm{e}^{\prime}(t)=(A-L G) e(t)+(B-L F)(\lambda(t)-\gamma(t)) \\
C e(t)+D(\lambda(t)-\gamma(t)) \in-\partial \Phi^{*--}(\lambda(t))+\partial \Phi^{*,-}(\gamma(t)) .
\end{array}\right.
$$

The proof of the asymptotic stability of (42) is similar to the proof of Theorem 4.4 in [11] and is omitted here.

In $[11,29]$ it is assumed that $D=0, \Phi(\cdot)=\psi_{K}(\cdot)$ for some closed convex set $K$, and $p \leq n$. However $y$ may depend explicitly on an absolutely continuous (resp. piecewise AC) function of time. Then $K=K(t)$ varies in an absolutely continuous way (resp. in a piecewise AC way allowing for state jumps) which is not the case in this paper. Hence the so-called extended observers (see [11, Eq.(7)]) cannot be designed relying on the above well-posedness results.

\section{Stability results}

Once the Lur'e system has been shown to be well-posed, one can study its stability properties. Let us set:

$$
X_{0}= \begin{cases}\left\{x \in \mathbb{R}^{n}: C_{I} x \in \operatorname{Dom}\left(\partial \Phi_{I}\right)\right\} & \text { if } p_{I} \geq 1 \\ \mathbb{R}^{n} & \text { if } p_{I}=0 .\end{cases}
$$

Then, for $x_{0} \in X_{0}$, problem $\operatorname{NSDS}\left(A, B, C, D, 0, \varphi_{1}, \ldots, \varphi_{p}, x_{0}\right)$ has a unique solution

$$
x\left(. ; x_{0}\right): \mathbb{R}_{+} \rightarrow \mathbb{R}^{n} ; t \mapsto \mathbb{R}^{n} .
$$

We note that problem $\operatorname{NSDS}\left(A, B, C, D, 0, \varphi_{1}, \ldots, \varphi_{p}, x_{0}\right)$ can be written as follows:

$$
\left\{\begin{array}{l}
(\text { a.e. } t \geq 0): x^{\prime}(t)=A x(t)+B \lambda(t) \\
x(0)=x_{0} \\
(\forall t \geq 0): y(t)=C x(t)+D \lambda(t) \\
(\forall t \geq 0): y(t) \in-\partial \Phi^{*,-}(\lambda(t))
\end{array}\right.
$$

and is denoted as the system $\left(A, B, C, D, \Phi^{*,-}\right)$. Let us state the following: 
Assumption 7. The initial data satisfy:

$0 \in \mathcal{X}_{0}$ and $\Lambda_{I I}(0) \in \partial \Xi_{I}(0)$.

Let us recall that the system in (43) may be written equivalently as the variational inequality:

$$
\left\langle X^{\prime}(t)-V A V^{-1} X(t)-\Lambda_{I I}(X(t)), v-X(t)\right\rangle+\Xi_{I}(v)-\Xi_{I}(X(t)) \geq 0, \quad \forall v \in \mathbb{R}^{n},
$$

with

$$
(\forall t \geq 0): X(t)=V x(t) .
$$

We see that Assumption 7 ensures that

$$
(\forall t \geq 0): x(t ; 0)=0 .
$$

In this section, we suppose that the Lur'e system is well-posed, i.e. Assumptions 1 through 4 or Assumptions 1 and 5 hold. We also suppose that Assumption 7 holds.

\subsection{Dissipativity and stability results}

We may now examine the question of stability of this last trivial solution. For this we shall rely on the property of dissipativity of dynamical systems, which plays a central role in control and feedback systems theory [30]. Let us first introduce several definitions of dissipative systems.

Definition 1. One says that the system $(A, B, C, D)$ is passive provided that there exists a symmetric and positive definite matrix $P \in \mathbb{R}^{n \times n}$ such that the matrix

$$
Q=\left(\begin{array}{cc}
A^{T} P+P A & P B-C^{T} \\
B^{T} P-C & -\left(D+D^{T}\right)
\end{array}\right)
$$

is negative semidefinite.

It is easy to show that the linear matrix inequality $Q \leq 0$ in (45) is equivalent to the so-called dissipation inequality:

$$
\frac{1}{2} x^{T}\left(t_{1}\right) \operatorname{Px}\left(t_{1}\right)-\frac{1}{2} x^{T}\left(t_{0}\right) \operatorname{Px}\left(t_{0}\right)=\int_{t_{0}}^{t_{1}} y^{T}(t) \lambda(t) \mathrm{d} t+\int_{t_{0}}^{t_{1}}\left(x^{T}(t) \lambda^{T}(t)\right) Q\left(\begin{array}{l}
x(t) \\
\lambda(t)
\end{array}\right) \mathrm{d} t
$$

for any $t_{1}, t_{0}, t_{1} \geq t_{0}$, see for instance Chapter 3 in [30]. One may also define the passivity with a positive semidefinite $P$. Then if the pair $(C, A)$ is observable it follows that the solutions of the LMI $Q \leq 0$ are full-rank, hence $P$ is positive definite [31].

Definition 2. One says that the system $(A, B, C, D)$ is strictly passive provided that there exists a symmetric and positive definite matrix $P \in \mathbb{R}^{n \times n}$ and a real $\varepsilon>0$ such that

$$
Q=\left(\begin{array}{cc}
A^{T} P+P A+\varepsilon P & P B-C^{T} \\
B^{T} P-C & -\left(D+D^{T}\right)
\end{array}\right)
$$

is negative semidefinite.

These two well-known definitions are now slightly extended to cope with the multivalued Lur'e systems we are dealing with.

Definition 3. One says that the system $\left(A, B, C, D, \Phi^{*,-}\right)$ is passive provided that there exists a symmetric and positive definite matrix $P \in \mathbb{R}^{n \times n}$ such that

$$
\left(\forall x \in \mathbb{R}^{n}, z \in \mathbb{R}^{p}\right):\langle P A x, x\rangle+\left\langle\left(P B-C^{T}\right) z, x\right\rangle-\langle D z, z\rangle+\Phi^{*,-}(0)-\Phi^{*,-}(z) \leq 0 .
$$

Definition 4. One says that the system $\left(A, B, C, D, \Phi^{*,-}\right)$ is strictly passive provided that there exists a symmetric and positive definite matrix $P \in \mathbb{R}^{n \times n}$ and a real $\varepsilon>0$ such that

$$
\left(\forall x \in \mathbb{R}^{n}, z \in \mathbb{R}^{p}\right):\left\langle P A x+\frac{1}{2} \varepsilon P x, x\right\rangle+\left\langle\left(P B-C^{T}\right) z, x\right\rangle-\langle D z, z\rangle+\Phi^{*,-}(0)-\Phi^{*,-}(z) \leq 0 .
$$

Let us note that $\left(A, B, C, D, \Phi^{*,-}\right)$ is strictly passive if and only if there exists $\varepsilon>0$ such that $\left(A+\frac{1}{2} \varepsilon I, B, C, D, \Phi^{*,-}\right)$ is passive. 
Proposition 4. If the system $(A, B, C, D)$ is passive and

$\left(\forall z \in \mathbb{R}^{p}\right): \Phi^{*,-}(z) \geq \Phi^{*,-}(0)$

then the system ( $\left.A, B, C, D, \Phi^{*,-}\right)$ is passive.

Proof. See the proof of Proposition 5 with $\varepsilon=0$.

Proposition 5. If the system $(A, B, C, D)$ is strictly passive and

$\left(\forall z \in \mathbb{R}^{p}\right): \Phi^{*,-}(z) \geq \Phi^{*,-}(0)$

then the system ( $\left.A, B, C, D, \Phi^{*,-}\right)$ is strictly passive.

Proof. Indeed,

$$
\begin{aligned}
& \left\langle P A x+\frac{1}{2} \varepsilon P x, x\right\rangle+\left\langle\left(P B-C^{T}\right) z, x\right\rangle-\langle D z, z\rangle+\Phi^{*,-}(0)-\Phi^{*,-}(z) \\
& \quad=\frac{1}{2}\left\langle\left(A^{T} P+P A\right) x+\varepsilon P x, x\right\rangle+\frac{1}{2}\left\langle\left[\left(P B-C^{T}\right)+\left(B^{T} P-C\right)\right] z, x\right\rangle-\frac{1}{2}\left\langle\left(D+D^{T}\right) z, z\right\rangle+\Phi^{*,-}(0)-\Phi^{*,-}(z) \\
& \quad=\frac{1}{2}\left(\begin{array}{ll}
x^{T} & \left.z^{T}\right) Q\left(\begin{array}{l}
x \\
z
\end{array}\right)+\Phi^{*,-}(0)-\Phi^{*,-}(z)
\end{array}\right.
\end{aligned}
$$

and the result holds.

Theorem 3. (1) If the system $\left(A, B, C, D, \Phi^{*,-}\right)$ is passive then there exists a constant $C>0$ such that for each $x_{0} \in X_{0}$ :

$(\forall t \geq 0):\left\|x\left(t ; x_{0}\right)\right\| \leq C\left\|x_{0}\right\|$.

(2) If the system ( $\left.A, B, C, D, \Phi^{*,-}\right)$ is strictly passive then there exist constants $C>0$ and $\alpha>0$ such that for each $x_{0} \in X_{0}$ :

$$
(\forall t \geq 0):\left\|x\left(t ; x_{0}\right)\right\| \leq C\left\|x_{0}\right\| \mathrm{e}^{-\alpha t} .
$$

Proof. Let $x_{0} \in X_{0}$ be given. We set $(\forall t \geq 0): x(t)=x\left(t ; x_{0}\right)$. Let us first prove part (2) of the theorem. We have:

$$
\begin{aligned}
x^{\prime}(t) & =A x(t)+B \lambda(t) \Rightarrow P x^{\prime}(t)=P A x(t)+P B \lambda(t) \\
& \Rightarrow\left\langle P x^{\prime}(t), x(t)\right\rangle=\langle P A x(t), x(t)\rangle+\left\langle\left(P B-C^{T}\right) \lambda(t), x(t)\right\rangle+\left\langle C^{T} \lambda(t), x(t)\right\rangle .
\end{aligned}
$$

The matrix $P$ is symmetric and positive definite and thus : $\left(\forall x \in \mathbb{R}^{n}\right):\langle P x, x\rangle \geq \lambda_{1}(P)\|x\|^{2}$ where $\lambda_{1}(P)>0$ is the smallest eigenvalue of the matrix $P$. We have

$$
\left\langle P x^{\prime}(t), x(t)\right\rangle=\frac{1}{2} \frac{\mathrm{d}}{\mathrm{d} t}\langle P x(t), x(t)\rangle .
$$

We have also $(\forall t \geq 0)$ :

$$
\begin{aligned}
y(t) & \in-\partial \Phi^{*,-}(\lambda(t)) \Rightarrow C x(t)+D \lambda(t) \in-\partial \Phi^{*,-}(\lambda(t)) \\
& \Rightarrow\langle C x(t)+D \lambda(t), v-\lambda(t)\rangle+\Phi^{*,-}(v)-\Phi^{*--}(\lambda(t)) \geq 0, \quad \forall v \in \mathbb{R}^{p} .
\end{aligned}
$$

Thus $(\forall t \geq 0)$ :

$$
-\langle C x(t), \lambda(t)\rangle-\langle D \lambda(t), \lambda(t)\rangle+\Phi^{*,-}(0)-\Phi^{*,-}(\lambda(t)) \geq 0
$$

and thus $(\forall t \geq 0)$

$$
\left\langle x(t), C^{T} \lambda(t)\right\rangle \leq-\langle D \lambda(t), \lambda(t)\rangle+\Phi^{*,-}(0)-\Phi^{*,-}(\lambda(t)) .
$$

It results that $(\forall t \geq 0)$ :

$$
\begin{aligned}
\frac{\mathrm{d}}{\mathrm{d} t}\langle P x(t), x(t)\rangle= & 2\left[\langle\operatorname{PAx}(t), x(t)\rangle+\left\langle\left(P B-C^{T}\right) \lambda(t), x(t)\right\rangle+\left\langle C^{T} \lambda(t), x(t)\right\rangle\right] \\
\leq & 2\left[\langle\operatorname{PAx}(t), x(t)\rangle+\left\langle\left(P B-C^{T}\right) \lambda(t), x(t)\right\rangle\right]-2\langle D \lambda(t), \lambda(t)\rangle+2 \Phi^{*,-}(0)-2 \Phi^{*,-}(\lambda(t)) \\
= & 2\left[\left\langle\operatorname{PAx}(t)+\frac{1}{2} \varepsilon x(t), x(t)\right\rangle+\left\langle\left(P B-C^{T}\right) \lambda(t), x(t)\right\rangle\right]-2\langle D \lambda(t), \lambda(t)\rangle \\
& +2 \Phi^{*,-}(0)-2 \Phi^{*,-}(\lambda(t))-\varepsilon\langle P x(t), x(t)\rangle \\
\leq & -\langle\varepsilon P x(t), x(t)\rangle \leq-\varepsilon \lambda_{1}(P)\|x(t)\|^{2} .
\end{aligned}
$$


Thus

$$
\langle P x(t), x(t)\rangle \leq\left\langle P x_{0}, x_{0}\right\rangle-\varepsilon \lambda_{1}(P) \int_{0}^{t}\|x(s)\|^{2} \mathrm{~d} s .
$$

Hence

$$
\|x(t)\|^{2} \leq \frac{\left\langle P x_{0}, x_{0}\right\rangle}{\lambda_{1}(P)}-\varepsilon \int_{0}^{t}\|x(s)\|^{2} \mathrm{~d} s .
$$

It results from Gronwall's lemma that

$$
\|x(t)\|^{2} \leq \frac{\left\langle P x_{0}, x_{0}\right\rangle}{\lambda_{1}(P)} \mathrm{e}^{-\varepsilon t} .
$$

Setting

$$
C=\sqrt{\frac{\|P\|}{\lambda_{1}(P)}}, \quad \alpha=\frac{\varepsilon}{2},
$$

we have proved that

$$
(\forall t \geq 0):\|x(t)\| \leq C\left|\left\|x_{0} \mid\right\| \mathrm{e}^{-\alpha t} .\right.
$$

The proof of part (1) of the theorem is obtained as above by setting $\varepsilon=0$.

Remark 8. (i) If the system $\left(A, B, C, D, \Phi^{*-}\right)$ is passive then for each $\epsilon>0$, there exists $\delta>0$ such that

$$
\left(x_{0} \in \mathcal{X}_{0},\left\|x_{0}\right\| \leq \delta\right) \Rightarrow(\forall t \geq 0):\left\|x\left(t ; x_{0}\right)\right\| \leq \epsilon .
$$

This relation ensures the stability of the trivial solution.

(ii) If the system $\left(A, B, C, D, \Phi^{*,-}\right)$ is strictly passive then the trivial solution is stable and

$$
\left(x_{0} \in X_{0}\right) \Rightarrow \lim _{t \rightarrow+\infty}\left\|x\left(t ; x_{0}\right)\right\|=0 .
$$

This last relation ensures the global attractivity of the trivial solution.

\subsection{An implication of dissipativity}

Suppose that the linear matrix inequality (LMI) in (45) is satisfied for some $P=P^{T}>0$, and that $D$ has the form in (6). Then using for instance the Schur complement Lemmas A.64 and A.65 in [30] it may be deduced that $D+D^{T} \geq 0$ and that $P B^{I}=C_{I}^{T}$ while

$$
Q=\left(\begin{array}{cc}
A^{T} P+P A & P B_{I I}-C_{I I}^{T} \\
\left(B^{I I}\right)^{T} P-C_{I I} & -\left(D_{I I}+D_{I I}^{T}\right)
\end{array}\right) \leq 0 .
$$

Therefore (12) holds with $W^{2}=P$. The application of the well-posedness results (and in particular whether Assumption 4 holds or not) then depends on whether the conditions of Propositions 1, 2 or 3 hold.

When the matrix $D$ is skew symmetric with $p_{I I}=p$ (see for instance (38)) then $D+D^{T}=0$ and one may again use Lemma A.64 in [30] to deduce that Assumption 5(i) holds.

\subsection{Comments}

The existence and uniqueness results, as well as the stability, are focused on the state $x(\cdot)$. The multiplier $\lambda(\cdot)$ is not considered. In practice however $\lambda$ may be a vector that contains physical variables (see Sections 3.1.4 and 3.2.2 with the circuits where $\lambda$ contains voltages and currents). It may then be better to consider the whole vector $\left(\begin{array}{l}x \\ \lambda\end{array}\right)$ into the wellposedness and the stability analysis. This is outside the scope of the present work, anyway.

\section{Invariance results}

In this section it is shown that the Krasovskii-LaSalle invariance principle extends to the autonomous multivalued Lur'e system under study (43). The first theorem concerns the continuity of the solutions with respect to the initial condition. We suppose that the Lur'e system is well-posed, i.e. Assumptions 1 through 4 or Assumptions 1 and 5 hold. We also suppose that Assumption 7 holds all through this section.

Theorem 4. If the system $(A, B, C, D)$ is passive then for each $t \geq 0$, the function

$$
x(t ; .): \chi_{0} \rightarrow \mathbb{R}^{n} ; x_{0} \mapsto x\left(t ; x_{0}\right)
$$

is Lipschitz continuous. 
Proof. Let $x_{1}^{0}, x_{2}^{0} \in X_{0}$ be given and set

$$
(\forall t \geq 0): x_{1}(t)=x\left(t ; x_{1}^{0}\right), \quad x_{2}(t)=x\left(t ; x_{2}^{0}\right) .
$$

Let also $\lambda_{1}(t)$ and $\lambda_{2}(t)$ be such that:

$$
C x_{1}(t)+D \lambda_{1}(t) \in-\partial \Phi^{*,-}\left(\lambda_{1}(t)\right)
$$

and

$$
C x_{2}(t)+D \lambda_{2}(t) \in-\partial \Phi^{*,-}\left(\lambda_{2}(t)\right)
$$

We have

$$
\left\langle P\left(x_{1}^{\prime}(t)-x_{2}^{\prime}(t)\right), x_{1}(t)-x_{2}(t)\right\rangle=\left\langle P A\left(x_{1}(t)-x_{2}(t)\right), x_{1}(t)-x_{2}(t)\right\rangle+\left\langle P B\left(\lambda_{1}(t)-\lambda_{2}(t)\right), x_{1}(t)-x_{2}(t)\right\rangle .
$$

Thus

$$
\begin{aligned}
& \frac{\mathrm{d}}{\mathrm{d} t}\left\langle P\left(x_{1}-x_{2}\right)(t),\left(x_{1}-x_{2}\right)(t)\right\rangle=2\left[\left\langle P A\left(x_{1}(t)-x_{2}(t)\right), x_{1}(t)-x_{2}(t)\right\rangle\right. \\
& \left.\quad+\left\langle\left(P B-C^{T}\right)\left(\lambda_{1}(t)-\lambda_{2}(t)\right), x_{1}(t)-x_{2}(t)\right\rangle+\left\langle C^{T}\left(\lambda_{1}(t)-\lambda_{2}(t)\right), x_{1}(t)-x_{2}(t)\right\rangle\right] .
\end{aligned}
$$

We have

$$
\left\langle C x_{1}(t)+D \lambda_{1}(t), v-\lambda_{1}(t)\right\rangle+\Phi^{*,-}(v)-\Phi^{*,-}\left(\lambda_{1}(t)\right) \geq 0, \quad \forall v \in \mathbb{R}^{p}
$$

and

$$
\left\langle C x_{2}(t)+D \lambda_{2}(t), v-\lambda_{2}(t)\right\rangle+\Phi^{*,-}(v)-\Phi^{*,-}\left(\lambda_{2}(t)\right) \geq 0, \quad \forall v \in \mathbb{R}^{p} .
$$

It results that

$$
\left\langle C x_{1}(t)+D \lambda_{1}(t), \lambda_{2}(t)-\lambda_{1}(t)\right\rangle+\Phi^{*,-}\left(\lambda_{2}(t)\right)-\Phi^{*,-}\left(\lambda_{1}(t)\right) \geq 0
$$

and

$$
\left\langle C x_{2}(t)+D \lambda_{2}(t), \lambda_{1}(t)-\lambda_{2}(t)\right\rangle+\Phi^{*,-}\left(\lambda_{1}(t)\right)-\Phi^{*,-}\left(\lambda_{2}(t)\right) \geq 0,
$$

from which we deduce that

$$
\left\langle C\left(x_{2}(t)-x_{1}(t)\right), \lambda_{2}(t)-\lambda_{1}(t)\right\rangle \leq-\left\langle D\left(\lambda_{2}(t)-\lambda_{1}(t)\right), \lambda_{2}(t)-\lambda_{1}(t)\right\rangle .
$$

Thus

$$
\begin{aligned}
& \frac{\mathrm{d}}{\mathrm{d} t}\left\langle P\left(x_{1}-x_{2}\right)(t),\left(x_{1}-x_{2}\right)(t)\right\rangle \leq 2\left[\left\langle P A\left(x_{1}(t)-x_{2}(t)\right), x_{1}(t)-x_{2}(t)\right\rangle\right. \\
& \left.\quad+\left\langle\left(P B-C^{T}\right)\left(\lambda_{1}(t)-\lambda_{2}(t)\right), x_{1}(t)-x_{2}(t)\right\rangle-\left\langle D\left(\lambda_{1}(t)-\lambda_{2}(t)\right), \lambda_{1}(t)-\lambda_{2}(t)\right\rangle\right] \leq 0 .
\end{aligned}
$$

Therefore

$$
\left\langle P\left(x_{1}(t)-x_{2}(t)\right), x_{1}(t)-x_{2}(t)\right\rangle \leq\left\langle P\left(x_{1}^{0}-x_{2}^{0}\right), x_{1}^{0}-x_{2}^{0}\right\rangle
$$

and

$$
\left\|x_{1}(t)-x_{2}(t)\right\| \leq \sqrt{\frac{\|P\|}{\lambda_{1}(P)}}\left\|x_{1}^{0}-x_{2}^{0}\right\| .
$$

For $x_{0} \in X_{0}$, we denote by $\gamma\left(x_{0}\right)$ the orbit

$$
\gamma\left(x_{0}\right):=\left\{x\left(\tau ; x_{0}\right) ; \tau \geq 0\right\},
$$

and by $\Lambda\left(x_{0}\right)$ the limit set

$$
\Lambda\left(x_{0}\right):=\left\{z \in \mathbb{R}^{n}: \exists\left\{\tau_{i}\right\} \subset[0,+\infty) ; \tau_{i} \rightarrow+\infty \text { and } x\left(\tau_{i} ; x_{0}\right) \rightarrow z\right\} .
$$

We say that a set $\mathscr{D} \subset X_{0}$ is invariant provided that

$$
x_{0} \in \mathscr{D} \Rightarrow \gamma\left(x_{0}\right) \subset \mathscr{D} \text {. }
$$

We also denote by $d(s, \mathcal{M})$ the distance from a point $s \in \mathbb{R}^{n}$ to a set $\mathcal{M} \subset \mathbb{R}^{n}$, that is $d(s, \mathcal{M}):=\inf _{m \in \mathcal{M}}\|s-m\|$. Taking advantage of the continuity property with respect to the initial conditions we may state the following:

Proposition 6. Assume that the system $(A, B, C, D)$ is passive. Let $x_{0} \in X_{0}$ be given. The set $\Lambda\left(x_{0}\right)$ is invariant. 
Proof. Let $z \in \Lambda\left(x_{0}\right)$ be given. There exists $\left\{\tau_{i}\right\} \subset[0,+\infty)$ such that $\tau_{i} \rightarrow+\infty$ and $x\left(\tau_{i} ; x_{0}\right) \rightarrow z$. Let $\tau \geq 0$ be given. Using Theorem 4 , we obtain $x(\tau ; z)=\lim _{i \rightarrow \infty} x\left(\tau ; x\left(\tau_{i} ; x_{0}\right)\right)$. Then remarking that $x\left(\tau ; x\left(\tau_{i} ; x_{0}\right)\right)=x\left(\tau+\tau_{i} ; x_{0}\right)$, we get $x(\tau ; z)=\lim _{i \rightarrow \infty} x\left(\tau+\tau_{i} ; x_{0}\right)$. Thus setting $w_{i}:=\tau+\tau_{i}$, we see that $w_{i} \geq 0, w_{i} \rightarrow+\infty$ and $x\left(w_{i} ; x_{0}\right) \rightarrow x(\tau ; z)$. It results that $x(\tau ; z) \in \Lambda\left(x_{0}\right)$.

Finally the result of the next lemma will be used in the invariance theorem:

Lemma 1. Assume that the system $(A, B, C, D)$ is passive. We set

$\left(\forall x \in \mathbb{R}^{n}\right): V(x)=\langle P x, x\rangle$.

Let $x_{0} \in \mathcal{X}_{0}$ be given. There exists a constant $k \in \mathbb{R}$ such that

$\left(\forall x \in \Lambda\left(x_{0}\right)\right): V(x)=k$.

Proof. Let us set $(\forall t \geq 0), x(t)=x\left(t ; x_{0}\right)$ and $V^{*}(t)=V(x(t))$. As it has been checked in the proof of Theorem 3, we have

$$
(\forall t \geq 0): \frac{\mathrm{d} V^{*}}{\mathrm{~d} t}(t)=2\left\langle P x^{\prime}(t), x(t)\right\rangle \leq 0 .
$$

It follows that $V^{*}(\cdot)$ is decreasing on $\left[0,+\infty\left[\right.\right.$. Moreover $V^{*}(\cdot)$ is bounded from below (by 0$)$ on $[0,+\infty$ [. It results that

$$
\lim _{\tau \rightarrow+\infty} V\left(x\left(\tau ; x_{0}\right)\right)=k
$$

for some $k \in \mathbb{R}$. Let $y \in \Lambda\left(x_{0}\right)$ be given. There exists $\left\{\tau_{i}\right\} \subset[0,+\infty)$ such that $\tau_{i} \rightarrow+\infty$ and $x\left(\tau_{i} ; x_{0}\right) \rightarrow y$. By continuity

$$
\lim _{i \rightarrow+\infty} V\left(x\left(\tau_{i} ; x_{0}\right)\right)=V(y) .
$$

Therefore $V(y)=k$. Here $y$ has been chosen arbitrary in $\Lambda\left(x_{0}\right)$ and thus

$$
\left(\forall y \in \Lambda\left(x_{0}\right)\right): V(y)=k .
$$

The invariance theorem for autonomous passive multivalued Lur'e systems (2) can now be stated:

Theorem 5 (Invariance Theorem). Assume that the system $(A, B, C, D)$ is passive. Assume also that:

$$
\left(\forall x \in \mathbb{R}^{p}, x \neq 0\right): \Phi^{*,-}(x)>\Phi^{*,-}(0) .
$$

Let $\mathcal{M}$ be the largest invariant subset of

$$
E=\left\{z \in \mathbb{R}^{n}:\langle P A z, z\rangle=0\right\} .
$$

Then for each $x_{0} \in \mathcal{X}_{0}$, the orbit $\gamma\left(x_{0}\right)$ is bounded and

$$
\lim _{\tau \rightarrow+\infty} d\left(x\left(\tau ; x_{0}\right), \mathcal{M}\right)=0 \text {. }
$$

Proof. Here $\left(A, B, C, D, \Phi^{*,-}\right)$ is passive since $(A, B, C, D)$ is passive and $\left(\forall x \in \mathbb{R}^{p}\right): \Phi^{*,-}(x) \geq \Phi^{*,-}(0)$ (see Proposition 4$)$. The set $\gamma\left(x_{0}\right)$ is thus bounded (see Theorem 3) and following standard arguments (see Remark 3.1(iii) and (iv) in [18]), we deduce that $\Lambda\left(x_{0}\right)$ is nonempty and

$$
\lim _{\tau \rightarrow+\infty} d\left(x\left(\tau ; x_{0}\right), \Lambda\left(x_{0}\right)\right)=0 .
$$

Let us now check that $\Lambda\left(x_{0}\right) \subset E$. From Lemma 1, there exists $k \in \mathbb{R}$ such that

$$
\left(\forall x \in \Lambda\left(x_{0}\right)\right): V(x)=k .
$$

Let $z \in \Lambda\left(x_{0}\right)$ be given. Using Proposition 6 , we see that $(\forall t \geq 0): x(t ; z) \in \Lambda\left(x_{0}\right)$ and thus

$$
(\forall t \geq 0): V(x(t ; z))=k .
$$

Thus

$$
\frac{\mathrm{d}}{\mathrm{d} t} V(x(t ; z))=0, \quad \text { a.e. } t \geq 0 .
$$

It results that for a.e. $t \geq 0$ :

$$
\langle P A x(t ; z), x(t ; z)\rangle+\langle P B \lambda(t ; z), x(t ; z)\rangle=0
$$


with

$C x(t ; z)+D \lambda(t ; z) \in-\partial \Phi^{*--}(\lambda(t ; z))$.

Thus

$$
\begin{aligned}
0 & =\langle P A x(t ; z), x(t ; z)\rangle+\left\langle\left(P B-C^{T}\right) \lambda(t), x(t ; z)\right\rangle+\left\langle C^{T} \lambda(t ; z), x(t ; z)\right\rangle \\
& \leq\langle P A x(t ; z), x(t ; z)\rangle+\left\langle\left(P B-C^{T}\right) \lambda(t ; z), x(t ; z)\right\rangle-\langle D \lambda(t ; z), \lambda(t ; z)\rangle+\Phi^{*,-}(0)-\Phi^{*,-}(\lambda(t ; z)) \\
& \leq \Phi^{*,-}(0)-\Phi^{*--}(\lambda(t ; z)) \leq 0 .
\end{aligned}
$$

It results that for a.e. $t \geq 0$ :

$$
\Phi^{*,-}(\lambda(t ; z))=\Phi^{*,-}(0)
$$

and then

$$
\lambda(t ; z)=0 .
$$

It results that for a.e. $t \geq 0$ and by continuity for all $t \geq 0$ :

$$
\langle P A x(t ; z), x(t ; z)\rangle=0 .
$$

Taking the limit as $t \rightarrow 0$, we obtain

$$
\langle P A z, z\rangle=0 .
$$

It results that $z \in E$. Finally $\Lambda\left(x_{0}\right) \subset \mathcal{M}$ since $\Lambda\left(x_{0}\right) \subset E$ and $\Lambda\left(x_{0}\right)$ is invariant.

Remark 9. Denoting by $\&$ the set of stationary solutions of our problem, i.e.

$$
s=\left\{z \in \mathbb{R}^{n}: A z+B \lambda=0 ; C z+D \lambda \in-\partial \Phi^{*,-}(\lambda)\right\} .
$$

It is clear that $\&$ is invariant and we check easily that the assumptions of Theorem 5 imply that

$$
s \subset E .
$$

Then if we can prove that $\delta$ is the largest invariant subset of $E$ then we may assert that for any $x_{0} \in X_{0}$, $\lim _{\tau \rightarrow+\infty} d\left(x\left(\tau ; x_{0}\right), \S\right)=0$.

Remark 10 (Feedback System). In the case $y=C x+D \lambda+F u$ and $f(t)=E u(t)$, where $u(\cdot)$ is some $m$-dimensional control input, then the study of this paper may be used to analyze feedback controllers of the form $u=S x+G \lambda$. It suffices to replace $(A, B, C, D)$ by $(A+E S, B+E G, C+F S, D+F G)$ in the analysis. For instance the condition (i) of Assumption 5 can be formulated as the problem:

P1: Find $P=P^{T}>0, P \in \mathbb{R}^{n \times n}$, and $S \in \mathbb{R}^{m \times n}, G \in \mathbb{R}^{m \times p}$ such that $P(B+E G)=(C+F S)^{T}$.

The feedback stabilization (resp. asymptotic stabilization) issue via dissipativity (Proposition 4 or 5 and Theorem 3 ) may then take the form of the nonlinear matrix inequality problem:

P2: Find $P=P^{T}>0, P \in \mathbb{R}^{n \times n}, S \in \mathbb{R}^{m \times n}$, and $G \in \mathbb{R}^{m \times p}$ such that

$$
\left(\begin{array}{ccc}
(A+E S)^{T} P+P(A+E S) & P(B+E G)-(C+F S)^{T} \\
(B+E G)^{T} P-(C+F S) & -(D+F G)-(D+F G)^{T}
\end{array}\right) \leq 0 \quad(\text { resp. }<0) .
$$

This problem reduces, if Problem $\mathbf{P} \mathbf{1}$ is solved with some $P$, to find $S$ and $G$ such that $(A+E S)^{T} P+P(A+E S) \leq 0$ and $-(D+F G)-(D+F G)^{T} \leq 0$. The fact that the nonlinear matrix inequality (48) possesses a solution relies on invertibility and minimum phase properties of the quadruple $(A, E, C, F)$ [30, Proposition 5.39].

\section{Conclusions}

In this paper a class of Lur'e systems with a multivalued feedback nonlinearity is studied. A non-zero feedthrough matrix is considered in the linear part. After some suitable transformations the system is proved to be, under certain conditions on the feedthrough matrix, well-posed using an extension of Kato's theorem. Another path to show the existence and uniqueness of solutions is based on maximal monotone operators, using a suitable state variable change. Stability properties related to dissipativity are studied and an extension of the Krasovskii-LaSalle invariance principle is proposed. Applications are in the study of electrical circuits with piecewise-linear electronic devices, and state observer design. A convergent timestepping method with order 1 or $\frac{1}{2}$ depending on the data is easily derived from [28]. 


\section{References}

[1] M.R. Liberzon, Essays on the absolute stability theory, Automation and Remote Control 67 (10) (2006) 1610-1644.

[2] B. Brogliato, Absolute stability and the Lagrange-Dirichlet theorem with monotone multivalued mappings, Systems and Control Letters 51 (5) (2004) 343-353.

[3] W.P.M.H. Heemels, J.M. Schumacher, S. Weiland, Linear complementarity systems, SIAM Journal on Applied Mathematics 60 (4) (2000) $1234-1269$.

[4] M.K. Camlibel, W.P.M.H. Heemels, A.J. van der Schaft, J.M. Schumacher, Switched networks and complementarity, IEEE Transactions on Circuits and Systems I 50 (8) (2003) 1036-1046.

[5] A.J. van der Schaft, J.M. Schumacher, The complementary-slackness class of hybrid systems, Mathematics of Control, Signals and Systems 9 (3) (1996) 266-301.

[6] D.M.W. Leenaerts, W.M.G. van Bokhoven, Piecewise Linear Modeling and Analysis, Kluwer, New York, 1998.

[7] L. Iannelli, F. Vasca, K. Camlibel, Complementarity and passivity for piecewise linear feedback systems, in: Proc. 45th IEEE Conf. on Decision and Control, San Diego, USA, 13-15 December 2006, pp. 4212-4217.

[8] D. Goeleven, B. Brogliato, Stability and instability matrices for linear evolution variational inequalities, IEEE Transactions on Automatic Control 49 (4) (2004) 521-534.

[9] B. Brogliato, D. Goeleven, The Krasovskii-LaSalle invariance principle for a class of unilateral dynamical systems, Mathematics of Control, Signals and Systems 17 (1) (2005) 57-76.

[10] B. Brogliato, A. Daniliidis, C. Lemaréchal, V. Acary, On the equivalence between complementarity systems, projected systems and differential inclusions, Systems and Control Letters 55 (1) (2006) 45-51.

[11] B. Brogliato, W.P.M.H. Heemels, Observer design for Lur'e systems with multivalued mappings: a passivity approach, IEEE Transactions on Automatic Control 54 (8) (2009) 1996-2001.

[12] J.C.A. de Bruin, A. Doris, N. van de Wouw, W.P.M.H. Heemels, H. Nijmeijer, Control of mechanical motion systems with non-collocation of actuation and friction: a Popov criterion approach for input-to-state stability and set-valued nonlinearities, Automatica 45 (2) (2009) 405-415.

[13] B. Cornet, Existence of slow solutions for a class of differential inclusions, Journal of Mathematical Analysis and Applications 96 (1983) $130-147$.

[14] C. Henry, An existence theorem for a class of differential equations with multivalued right-hand side, Journal of Mathematical Analysis and Applications 41 (1973) 179-186.

[15] P. Dupuis, A. Nagurney, Dynamical systems and variational inequalities, Annals of Operations Research 44 (1-4) (1993) 9-42.

[16] B. Brogliato, L. Thibault, Existence and uniqueness of solutions for non-autonomous complementarity dynamical systems, Journal of Convex Analysis $17(3-4)(2010)$.

[17] P.D. Panagiotopoulos, Inequality Problems in Mechanics and Applications, Birkhäser, Boston, 1985

[18] S. Adly, D. Goeleven, A stability theory for second-order nonsmooth dynamical systems with application to friction problems, Journal de Mathématiques Pures et Appliquées 83 (1) (2004) 17-51.

[19] M.K. Camlibel, W.P.M.H. Heemels, J.M. Schumacher, Consistency of a time-stepping method for a class of piecewise-linear networks, IEEE Transactions on Circuits and Systems I 49 (2002) 349-357.

[20] M.K. Camlibel, W.P.M.H. Heemels, J.M. Schumacher, On linear passive complementarity systems, European Journal of Control 8 (3) (2002) $220-237$.

[21] R.T. Rockafellar, R.J.B. Wets, Variational Analysis, in: Grundlehren der Mathematischen Wissenschaften, vol. 317, Springer, 1998.

[22] K. Addi, B. Brogliato, D. Goeleven, A qualitative mathematical analysis of a class of linear variational inequalities via semi-complementarity problems: applications in electronics, Mathematical Programming A, in press, doi:10.1007/s10107-009-0268-7.

[23] R.W. Cottle, J.-S. Pang, R.E. Stone, The Linear Complementarity Problem, Academic Press, 1992.

[24] D. Goeleven, D. Motreanu, V.V. Motreanu, On the stability of stationary solutions of first order evolution variational inequalities, Advances in Nonlinear Variational Inequalities 6 (2003) 1-30.

[25] K. Addi, S. Adly, B. Brogliato, D. Goeleven, A method using the approach of Moreau and Panagiotopoulos for the mathematical formulation of nonregular circuits in electronics, Nonlinear Analysis: Hybrid Systems 1 (1) (2007) 30-43.

[26] V. Acary, B. Brogliato, Numerical Methods For Nonsmooth Dynamical Systems Applications in Mechanics and Electronics, in: LNACM, vol. 35, Springer Verlag, 2008.

[27] J. Bastien, C.H. Lamarque, Persoz's gephyroidal model described by a maximal monotone differential inclusion, Archives Applied Mechanics 78 (2008) 393-407.

[28] J. Bastien, M. Schatzman, Numerical precision for differential inclusions with uniqueness, Mathematical Modelling and Numerical Analysis (ESAIM:M2AN) 36 (3) (2002) 427-460.

[29] B. Brogliato, W.P.M.H. Heemels, Design of observers and certainty equivalence controllers for differential inclusions: a passivity approach, INRIA Research Report RR-6916, 2009, http://hal.inria.fr/inria-00380820/fr/.

[30] B. Brogliato, R. Lozano, B. Maschke, O. Egeland, Dissipative Systems Analysis and Control. Theory and Applications, 2nd ed., Springer Verlag, 2007.

[31] M.K. Camlibel, L. Iannelli, F. Vasca, Passivity and complementarity, submitted to Mathematical Programming A, 2009 (in press) 